Cahiers de recherche sociologique

\title{
La collaboration avec la recherche universitaire vue de l'entreprise. Quelques résultats d'enquêtes dans les secteurs des technologies optoélectroniques
}

\author{
Jean-Paul Beltramo
}

Numéro 40, 2005

L’économie du savoir : une économie de la collaboration?

URI : https://id.erudit.org/iderudit/1002420ar

DOI : https://doi.org/10.7202/1002420ar

Aller au sommaire du numéro

Éditeur(s)

Département de sociologie - Université du Québec à Montréal

ISSN

0831-1048 (imprimé)

1923-5771 (numérique)

Découvrir la revue

Citer cet article

Beltramo, J.-P. (2005). La collaboration avec la recherche universitaire vue de l'entreprise. Quelques résultats d'enquêtes dans les secteurs des technologies optoélectroniques. Cahiers de recherche sociologique, (40), 111-169.

https://doi.org/10.7202/1002420ar
Résumé de l'article

Analysant les caractéristiques de la demande de connaissances externes des entreprises en fonction de leurs régimes d'innovation, l'article s'intéresse plus particulièrement au rôle donné à la recherche universitaire dans les stratégies d'innovation des entreprises. Les résultats tirés d'enquêtes auprès d'entreprises britanniques et françaises, développant des composants optoélectroniques, mettent en évidence l'existence de deux catégories de recherche : la R-D sur financement propre qui est liée aux objectifs à court terme, et la R-D à moyen et long terme qui dépend du financement externe. La participation de la recherche universitaire à la recherche à court terme de l'entreprise prend la forme principale de prestations de services, tandis que sa contribution à la recherche à long terme se réalise essentiellement par la mise à disposition contractuelle de doctorants. De plus, les collaborations avec les universités ne semblent pas avoir d'effets directs sur l'organisation de l'innovation à l'intérieur de la firme. 


\section{La collaboration avec la recherche universitaire vue de l'entreprise. Quelques résultats d'enquêtes dans les secteurs des technologies optoélectroniques}

Jean-Paul BELTRAMO

\section{Introduction}

L'analyse des interactions entre la science et l'industrie se focalise souvent sur la transformation de la production des savoirs qui peut en résulter ou, dans une perspective normative, sur les évolutions nécessaires à une meilleure adaptation des institutions scientifiques aux besoins des entreprises. Des indicateurs attestent que les normes du marché ont tendance à se développer au sein de la recherche publique: accroissement du nombre de dépôts de brevets par les universités, augmentation du financement contractuel donc de la recherche à court terme ayant des implications commerciales, avec pour conséquence le risque d'abandon des domaines où les perspectives de profit sont faibles dans l'immédiat'. En

1. Si la commercialisation des résultats de la recherche universitaire s'est accrue, elle n'en constitue pas moins une part fort modeste du financement des universités (les revenus de propriété intellectuelle des universités américaines, par exemple, représentent moins de $1 \%$ de leur budget total: P. Malissard, Y. Gingras et B. Gemme, «La commercialisation de la recherche», Actes de la recherche en sciences sociales, $\mathrm{n}^{\circ} 148$, juin 2003, p. 57-67. C'est d'ailleurs un argument supplémentaire pour considérer que la théorisation sur «le Mode 2», comme nouveau mode de production des connaissances, est plus un projet politique qu'une analyse de la réalité présente. Voir, pour la présentation de la «nouvelle production des savoirs»: M. Gibbons, H. Novotny, C. Limoges, S. Schwartzman, P. Scott et M. Trow, The New Production of Knowledge: The dynamics of science and research in contemporary societies, Londres, Sage, 1994. Pour les critiques, on peut se référer à: P. Weingart, «From "Finalization" to "Mode 2": old wine in new bottles?», Social Science Information, vol. 36, n 4,1997, p. 591 - 
revanche, on a beaucoup moins d'informations concernant l'effet de la coopération sur l'organisation de la recherche industrielle et, au-delà, sur les stratégies et la gestion de l'innovation au sein des entreprises. L'idée d'une interpénétration symétrique des mondes universitaire et industriel est rien moins qu'évidente. Si des chercheurs industriels publient, cosignent des articles avec des universitaires et participent à des congrès scientifiques $^{2}$, globalement, l'évolution des régimes d'innovation au sein des entreprises ne va pas dans le sens d'une pénétration des normes universitaires: après une période de relative autonomisation des activités de recherche et développement (R-D), illustrée par la création des laboratoires centraux au sein des grandes entreprises, un mouvement en sens inverse semble se dessiner qui voit une intégration plus poussée de la fonction de R-D aux autres fonctions de l'entreprise et sa subordination à la logique financière.

En s'appuyant sur les résultats d'enquêtes en entreprises, et sur la base d'une analyse de la variété des collaborations technologiques qui accompagnent la diversité des régimes d'innovation internes observés, il s'agit d'essayer de préciser la place occupée par les dispositifs d'accès aux connaissances universitaires dans les stratégies d'innovation des entreprises. L'objectif est également d'illustrer le comportement des entreprises vis-à-vis de l'évolution des «infrastructures de connaissances ${ }^{3}$ » et des incitations à la coopération mises en œuvre par les politiques publiques.

613; D. Pestre, «La production des savoirs entre académies et marché - Une relecture historique du livre «The new production of knowledge» édité par M. Gibbons», Revue d'Économie Industrielle, n' ${ }^{\circ} 79,1997$, p. 163-174; B. Godin, «Writing performative history: the new New Atlantis?», Social Studies of Science, vol. 28, n ${ }^{\circ} 3,1998$, p. $465-$ 483; T. Shinn, «Change or Mutation? Reflections on the Foundations of Contemporary Science", Social Science Information, vol. 38, n'1, mars 1999, p. 149-176; B. Godin et $\mathrm{Y}$. Gingras, «The place of universities in the system of knowledge production», Research Policy, vol. 29, 2000, p. 273-278; T. Shimn, «Nouvelle production du savoir et triple hélice», Actes de la recherche en sciences sociales, 141-142, mars 2002, p. 21-30.

2. La nouveauté du phénomène doit également être relativisée. En 1920 par exemple, les physiciens travaillant dans les laboratoires industriels représentent un quart des membres de l'American Physical Society, et 22\% des articles publiés dans Physical Review proviennent de ces mêmes laboratoires (contre $2 \%$ en 1910 et $14 \%$ en 1915 ). D. Pestre, «Louis Néel, le magnétisme et Grenoble», Cahiers pour l'histoire du CNRS, $\mathrm{n}^{\circ}$ 8, Éditions du CNRS, 1990, à partir des chiffres de P. K. Hoch, «The rise of Physics Laboratories in the Electrical Industry», Phys. Technol., 16, 1985, p. 177-183.

3. L'expression est emprunté à K. Smith, «Economic infrastructure and innovations systems», dans C. Edquist, Systems of innovation: Technology Institutions and Organizations, Londres, Pinter, 1997, p. 86-106. 


\section{Références conceptuelles et hypothèses}

Les innovations technologiques sont des éléments constitutifs du processus de production et, pour la majorité d'entre elles, on peut suivre le point de vue évolutionniste qui considère que dans une situation de complexité et d'incertitude, donc de rationalité limitée, elles procèdent de «comportements routiniers» des organisations ${ }^{4}$. Les routines mises en œuvre par la firme pour la production et l'innovation représentent également la forme principale d'accumulation de ses connaissances opérationnelles spécifiques. Si l'apprentissage par la pratique est un trait essentiel de l'acquisition des compétences de la firme, que cet apprentissage soit interactif est non moins important. Le rôle clé pour l'innovation des relations entre utilisateurs et producteurs a été souligné ${ }^{5}$, comme simple échange d'informations sur les occasions technologiques et sur les besoins des consommateurs, ou sous la forme d'une coopération plus directe pour la recherche d'une solution adaptée à un problème spécifique. La fonction de conception, entendue comme une adaptation du produit aux besoins des utilisateurs et aux contraintes de la production, tend à occuper une place centrale dans les analyses des processus d'innovation. Non seulement l'entreprise se réorganise autour d'une démarche de conception orientée vers l'innovation, mais la conception serait aussi la clé des coopérations entre les entreprises ${ }^{6}$. La conception modulaire de la fabrication d'un produit ou d'un système, où chaque unité productive fournit un composant dont la fonction et les contraintes d'interfaçage ont été spécifiées, permet de répondre à l'impératif de flexibilité qui caractérise les nouvelles conditions de la concurrence. Le système modulaire peut être mis en œuvre au sein de l'entreprise mais aussi dans un cadre de coopérations interentreprises (ou de réseaux d'entreprises); dans ce cas, l'intégration organisationnelle au sein d'un projet piloté par une grande entreprise multinationale mondialisée oblige chaque partenaire à se réorganiser en fonction de la conception du projet.

La mise en cuvre de l'innovation relève de l'activité des entreprises, de leurs collaborations et d'une façon générale de l'organisation du sys-

4. R. Nelson et S. Winter, An Evolutionary Theory of Economic Change, Cambridge, The Belknap Press of Harvard University Press, 1982.

5. B. A. Lundvall, Product Innovation and User-Producer Interaction, Aalborg University Press, 1985.

6. D. Encaoua et al., Les enjeux économiques de l'innovation, Bilan scientifique du programme CNRS, 2001. 


\section{L'économie du savoir: une économie de la collaboration?}

tème productif. Cela ne signifie pas que toutes les sources de l'innovation soient endogènes au système productif. Au sein même de la firme, des investissements (dont certains peuvent être qualifiés d'intangibles ou d'immatériels) sont consacrés aux activités génératrices de changement: conception, organisation, formation du personnel, contrôle-qualité, expertise, R-D, essais ${ }^{7}$... Les firmes peuvent même avoir des connaissances qui dépassent leurs compétences de base et qu'elles peuvent valoriser. Par ailleurs, l'histoire des temps modernes a montré comment les résultats de la science étaient susceptibles de créer des occasions de changements technologiques considérables ${ }^{8}$. Le développement des accords de recherche entre les entreprises et les universités et les laboratoires publics est une des formes d'appropriation des résultats scientifiques et technologiques.

La nécessité de l'apprentissage est aussi associée à l'acquisition des connaissances externes. Dans cette perspective, un ensemble de travaux, dont l'article de référence de 1989 de W. M. Cohen et D. A. Levinthal', a illustré l'existence d'une double fonction de la R-D de la firme, de production de connaissances internes et d'absorption de connaissances externes. Le modèle de Cohen et Levinthal établit une relation quantitative entre les investissements de R-D de la firme et sa capacité d'absorption des connaissances externes disponibles, qu'elles proviennent des autres entreprises, des universités ou des laboratoires publics. En revanche, il ne rend pas compte des liens technologiques entre clients et fournisseurs, ou plus largement interentreprises, non médiatisés par les départements de recherche mais suscités par la conception des produits ou des systèmes, tels qu'ils ont été évoqués plus haut. La démarche qui consiste à relier le niveau d'activité interne de R-D et l'assimilation des connaissances externes semble avoir plus de solidité quand elle s'applique aux seules connaissances scientifiques, en pratique aux relations avec la recherche universitaire. Le choix entre une stratégie de R-D contractuelle et une stratégie de R-D intégrée serait plus libre pour les relations interentreprises. Le postulat de la complémentarité des activités de R-D quand il s'agit des

7. P. Patel et K. Pavitt, «Nature et importance des systèmes nationaux d'innovations», STI Revue, n 14, OCDE, 1994.

8. La transformation des connaissances technologiques nouvelles en produits ou procédés nouveaux nécessite un travail de conception spécifique qui généralement fait appel à d'autres connaissances. Les innovations radicales qui peuvent être issues de la science feraient ainsi l'objet d'un certain «comportement routinien», comme les innovations incrémentales.

9. W. M. Cohen et D. A. Levinthal, «Innovation and Learning: The Two Faces of R.-D.», The Economic Journal, vol. 99, septembre 1989, p. 569-596. 
tenaires ${ }^{14}$. L'utilisation de la notion de «recherche partagée», hors du contexte des biotechnologies, semble plutôt correspondre à un classique transfert de connaissances, le partage ne concernant que les moyens apportés par chacun des partenaires, généralement les ressources humaines par le laboratoire universitaire ou le laboratoire public, et le financement par l'entreprise ${ }^{15}$. Au sein même du secteur pharmaceutique, les grands groupes se positionnent plutôt comme des gestionnaires de projets utilisant des connaissances produites en dehors de la firme par des partenaires. Ces pratiques s'inscrivent dans un mouvement de désengagement d'activités jugées non stratégiques ${ }^{16}$ et de redéfinition des compétences de la firme:

Enfin, les grands groupes pharmaceutiques apparaissent de plus en plus comme des intégrateurs de réseaux, qui externalisent en amont leur recherche, et qui externaliseront sans doute à terme leur production (à l'exception peut-être de certains blockbusters ${ }^{17}$ ). Leur compétence stratégique semble de plus en plus être celle de préempter les bons partenaires dans un ensemble relativement réduit, d'évaluer les technologies émergentes les plus prometteuses, et de limiter les externalités qui profiteraient à leurs concurrents directs ${ }^{18}$.

La recherche partagée, au sens strict d'équipes mixtes travaillant sur le même projet, est loin de constituer le mode principal de coopération entre

14. M. Cassier et D. Foray, «L'économie des consortia de haute technologie: études de cas dans la recherche biomédicale», Collection Les Cahiers de l'Innovation, $\mathrm{n}^{\circ} 99059$, CNRS, 1999.

15. M.-P. Bès et M. Grossetti, «Les apprentissages relationnels et cognitifs dans les relations entre les laboratoires du CNRS et les entreprises - le cas des sciences pour l'ingénieur", Rapport pour le programme Les enjeux économiques de l'innovation du CNRS, Cers - Université de Toulouse le Mirail, 2003, p. 8.

16. Le recentrage sur la définition du produit et sur les étapes de sa production et de sa commercialisation pour lesquelles l'entreprise a des compétences spécifiques touche la plupart des industries. Voir par exemple, M. Delapierre, «Globalisation et rôle stratégique accru de la connaissance: vers l'émergence de nouvelles formes d'oligopoles") Colloque Technologie et connaissance dans la mondialisation, Université de Poitiers, 9-11 septembre 1998.

17. Médicament dont le chiffre d'affaire annuel unitaire dépasse le milliard de dollars.

18. A. Bureth, «Nature composite de la connaissance et dynamiques d'innovation du secteur pharmaceutique: les enjeux de l'interface Universités-Entreprises», document préparé pour le Séminaire INRA-ENESAD, Dijon, 13 octobre 2003, p. 15. 
relations entre l'entreprise et la recherche publique, et de la substituabilité des activités de R-D quand il s'agit des relations entre les entreprises, suppose également l'existence d'une différence marquée entre les deux types de collaborations. Cette distinction peut prendre appui sur le modèle de S. J. Kline et N. Rosenberg ${ }^{10}$ qui stylise les processus d'innovation au sein des entreprises et leurs liens avec les sources externes de connaissances. Les auteurs indiquent que les connaissances nécessaires au début du processus sont généralement différentes de celles qui sont nécessaires aux différentes étapes de la chaîne d'innovation: au début, il est plutôt fait appel à la recherche à long terme alors qu'au cours du procès de développement, les recherches concernent plus les innovations de procédés et les analyses de systèmes. La séparation opérée entre, d'une part, les collaborations technologiques interentreprises et, d'autre part, les relations entre les entreprises et la recherche, procède également de la reconnaissance de la différenciation fonctionnelle de la production de connaissances scientifiques attestée par la persistance des disciplines scientifiques au-delà des problèmes à résoudre ${ }^{11}$ et de l'existence d'un mode spécifique de production scientifique institué selon ses propres règles, de divulgation en particulier ${ }^{12}$.

Une autre façon de concevoir les coopérations scientifiques et technologiques est d'en faire des arrangements institutionnels susceptibles de coproduire des connaissances, dans l'optique des thèses, évoquées plus haut, sur le nouveau mode de production des savoirs, dont les produits comme les lieux de production seraient des hybrides ${ }^{13}$. Des études réalisées dans le secteur de l'industrie pharmaceutique, et des biotechnologies en particulier, identifient les structures de coopération entre la recherche publique et la recherche privée comme des lieux de production de biens collectifs, c'est-à-dire de connaissances partagées par un réseau de par-

10. S. J. Kline et N. Rosenberg, «An Overview of Innovation», dans National Academy of Engineering, The Positive Sum Strategy: Harnessing Technology for Economic Growth, Washington, The National Academy Press, 1986, p. 275-305.

11. P. Weingart, op. cit.

12. P. Dasgupta et P. A. David, «Information Disclosure and the Economics of Science and Technology", dans G. Feiwel (dir.), Arrow and the ascent of modern economic theory, New York, New York University Press, 1987; P. Dasgupta et P. A. David, «Toward a new economics of science», Research Policy, vol. 23, $\mathrm{n}^{\circ} 5,1994$, p. $487-$ 521.

13. M. Cassier, «Compromis institutionnels et hybridations entre recherche publique et recherche privée», Revue d'Économie Industrielle, $\mathrm{n}^{\circ} 79,1997, \mathrm{p} .191-212$. 
la recherche privée et la recherche publique, y compris au sein des laboratoires mixtes ${ }^{19}$. En revanche, le développement peut être réellement partagé par les entreprises, mais se pose alors la question du secret (y compris entre clients et fournisseurs qui peuvent aussi être des concurrents).

Les observations sur le champ d'application très limité des processus d'hybridation de la recherche publique et de la recherche privée confortent notre postulat sur le caractère non substitutif, en règle générale, des relations technologiques interentreprises, d'une part, et des liaisons entre les entreprises et la recherche universitaire, d'autre part. L'intensité de l'innovation de l'entreprise ne serait pas liée de façon univoque à l'intensité de la coopération avec les universités. L'arbitrage entre R-D interne et R-D en coopération serait plus contraint avec la recherche publique qu'avec les autres entreprises, la recherche partagée intervenant essentiellement dans ce dernier cas. Le cadre d'analyse proposé ne se limite pas à une relation unique entre l'intensité de la R-D (ou la propension à innover) et la propension à coopérer, mais met en rapport la R-D interne avec les diverses sources de connaissances externes. La R-D interne est saisie à la fois par son intensité et par son organisation, tout au moins par un élément simple, l'existence ou non d'un centre de recherche au sein du groupe et la nature des relations entre le centre de R-D et l'unité de production considérée. La question complexe des liens entre le modèle organisationnel de la firme, la diversité de ses stratégies de partenariat et la complémentarité des ressources est ainsi abordée, avec, comme question corollaire, l'impact des caractéristiques technologiques sectorielles et la variété des stratégies individuelles des entreprises. La nature de notre base de données ne permet pas de pousser plus loin l'esquisse de formalisation; en revanche les entretiens réalisés auprès des entreprises fournissent des informations qualitatives complémentaires, en particulier sur les formes des collaborations.

De façon complémentaire, on s'interroge sur les évolutions en cours et leurs conséquences sur les relations entre les entreprises et la recherche. La préférence donnée aux comportements routiniers pour innover, plutôt qu'à des investissements risqués pour une recherche aux résultats incertains, la subordination des activités de R-D aux activités de conception des produits

19. C'est tout au moins ce qu'il ressort d'une enquête exploratoire que nous avons effectuée auprès de quelques laboratoires communs à des entreprises et à des organismes publics ou des universités. Voir aussi, T. Shinn, «Change or Mutation?...», op. cit. 
et des procédés (elles-mêmes soumises aux stratégies commerciales et financières), l'intégration du développement au niveau de la production modulaire des composants du produit comme réponse flexible à la concurrence, l'externalisation et le partage de la R-D dans le cadre d'accords technologiques, sont quelques-uns des arguments qui peuvent être avancés pour expliquer des changements majeurs dans l'organisation de la recherche industrielle. Un certain nombre de grands laboratoires d'entreprises, dont les prestigieux Laboratoires Bell, ont effectivement fait l'objet de démantèlement. Les transformations de la recherche industrielle ne sont pas uniformes, ne serait-ce qu'en raison de la très inégale répartition de la $\mathrm{R}-\mathrm{D}$, entre les secteurs industriels et selon la taille des entreprises. Mais audelà de la diversité des évolutions, c'est la redéfinition de la fonction de recherche au sein des entreprises qui est posée. L'investissement dans l'entretien d'une capacité d'absorption de la recherche externe n'apparaît plus comme stratégique, la priorité étant donnée à l'intégration de la recherche à la conception des produits et des procédés. L'utilisation de la veille technologique et des alliances stratégiques de R-D pour acquérir des connaissances scientifiques et technologiques comme substitut à la création de nouvelles capacités internes de recherche a été évoquée, en particulier, à propos des grandes multinationales de la pharmacie. Mais en externalisant sa recherche, l'entreprise diminuerait contradictoirement sa capacité à bénéficier de la recherche externe. Une des limitations de la demande de coopération des entreprises vis-à-vis de la recherche universitaire pourrait venir de cette contradiction, si, comme nous l'avons évoqué, les collaborations de recherche représentent plutôt des transferts de connaissances que des productions conjointes de connaissances ${ }^{20}$.

\section{Matériaux empiriques}

Notre base empirique principale est constituée par la nouvelle exploitation d'une partie des entretiens réalisés en entreprise en 1997 dans le

20. D. Teece a attiré l'attention sur les conséquences négatives d'une autre forme d'externalisation, celle qui concerne certaines activités de production, en raison de la distance créée entre la recherche et la fabrication: «Relatedly, managerial decisions to "hollow out" the corporation by out-sourcing components and other subsystems may have the indirect effect of impairing the innovation process by establishing barriers to the transfer of information between research and manufacturing, possibly causing future designs to be less sensitive to manufacturability concerns», D. Teece, «Technological change and the nature of the firm», dans G. Dosi et al. (dir.), Technical Change and Economic Theory, Londres, Pinter, 1990, p. 277. 
cadre d'une étude comparative franco-britannique ${ }^{21}$. L'enquête porte sur les modalités d'accès aux connaissances externes de firmes localisées en France et en Grande-Bretagne qui fabriquent des produits ou des systèmes optoélectroniques. Les entretiens ont été réalisés auprès des directions générales et des directions technologiques des établissements de production et des centres de R-D des entreprises ainsi qu'auprès de responsables d'entreprises de services d'études et de recherches, de laboratoires universitaires, de laboratoires publics et de diverses structures de transferts de technologies. Les informations traitées ici sont pour l'essentiel extraites des entretiens effectués dans les établissements de production. Ce choix correspond à la perspective qui consiste à situer le recours à la recherche publique dans le cadre des objectifs industriels.

On entend généralement par optoélectronique un ensemble de sciences et de technologies dont l'intérêt dominant est la conception et la fabrication de composants pour fibres optiques, lasers et systèmes de détection. Les composants optoélectroniques de base tels que les lasers sont réalisés à partir de matériaux semi-conducteurs. Les applications optoélectroniques concernent, dans un ordre décroissant: les télécommunications, les équipements militaires et aérospatiaux, les technologies de l'information et diverses autres industries telles que l'instrumentation scientifique et médicale, la métrologie, etc., avec une mention particulière pour le secteur de l'automobile, où, dans l'avenir, les fonctions optoélectroniques devraient prendre une part prépondérante dans l'équipement des véhicules ${ }^{22}$.

La physique des lasers et des dispositifs optoélectroniques s'inscrit parmi les disciplines universitaires au sein de la physique du rayonnement et de la matière. On peut aussi considérer que l'optoélectronique est un domaine de l'optique qui a contribué au renouvellement de cette discipline.

21. Cette enquête est le résultat d'une collaboration entre l'Institut de recherche en économie de l'éducation (IREDU), unité mixte de recherche du CNRS et de l'Université de Bourgogne, et le National Institute of Economic and Social Research (NIESR) de Londres. Voir le rapport: G. Mason, J.-P. Beltramo et J. J. Paul, High-level skills, knowledge infrastructure and industrial performance: electronics in Britain and France, NIESR, Londres IREDU-CNRS, Dijon, janvier 2000. Alors que dans cette étude, l'accent a été mis sur le rôle de la main-d'œuvre hautement qualifiée pour les échanges de recherche et sur la comparaison des systèmes de formation supérieure et d'innovation en France et en Grande-Bretagne, la nouvelle exploitation est centrée sur la diversité des sources de connaissances et sur les stratégies des entreprises pour la recherche de solutions aux problèmes posés par l'innovation.

22. Yole Développement et Thalès Airborne Systems, Étude générique sur les technologies optoélectroniques, Ministère de l'Économie, des Finances et de l'Industrie, Paris, juin 2002. 
La nouvelle optique, dénommée aussi photonique, embrasse le domaine d'intervention des photons, intégrant les avancées de plusieurs disciplines, notamment de la physique du solide, de la science des matériaux et du traitement de l'information ${ }^{23}$. Comme il y a l'électronique, science et industrie de l'électron, de façon complémentaire se constituerait la photonique, science et industrie du photon ${ }^{24}$. Depuis la conception du premier laser à rubis par le physicien américain Theodore Mainman en 1960, le développement des technologies optoélectroniques est allé de pair avec les avancées de la physique des lasers et de l'optique, sur la base d'apports réciproques $^{25}$. Est-on pour autant face à un nouveau régime d'innovation dans les secteurs industriels utilisant les technologies optoélectroniques qui s'apparenterait au modèle du secteur des bio-technologies et de la pharmacie, souvent décrit comme fondé sur la connaissance?

L'intérêt du choix de l'optoélectronique comme terrain d'enquête nous paraît reposer sur le fait qu'il s'agit d'une nouvelle technologie, mais avec des caractéristiques de produits et de marchés a priori très différentes de celles des biotechnologies, par ailleurs beaucoup plus étudiées. D'une part, si la connaissance scientifique est la ressource stratégique qui oriente les activités de marché des biotechnologies, c'est d'abord parce que la valeur du médicament ne réside pas dans sa technique de production mais dans le principe actif qui en est à l'origine, alors que, a contrario, la question de l'industrialisation est centrale pour la conception des composants optoélectroniques. D'autre part, contrairement aux marchés des biotechnologies, les marchés des composants optoélectroniques ont connu des crises importantes.

23. Comité des Applications de l'Académie des Sciences, De l'optique à la photonique, Rapport n ${ }^{\circ} 11$, Académie des Sciences, Paris, 1989. La photonique peut être entendue comme une optique appliquée dans la mesure où ce sont les nouveaux champs d'application de l'optique qui sont à l'origine de la nouvelle dénomination.

24. La photonique a une interface avec l'électronique, mais tend aussi à être une alternative à l'électronique, dans les télécommunications comme dans le traitement de l'information ou dans le biomédical par exemple; par rapport au terme générique de photonique, l'usage du terme optoélectronique correspond à un intérêt plus spécifique pour les semi-conducteurs.

25. Le premier laser à semi-conducteur a été inventé en 1962 et c'est à partir de la réalisation du laser à hétérojonction en 1972, permettant un fonctionnement à température ambiante, que l'on a commencé à maîtriser l'émission, la propagation et la réception de la lumière pour des usages industriels et militaires ainsi que dans le domaine médical, l'emploi de la fibre optique pour la transmission des informations devenant effectif avec les années 1980 . 
Les caractéristiques sectorielles ne déterminent pas nécessairement les structures d'innovation et de coopération scientifique et technologique de chaque entreprise. La comparaison de dix-sept établissements dont les produits principaux sont tous classés parmi les composants et les systèmes optoélectroniques permet d'analyser la variété stratégique au sein d'un même secteur. La localisation des établissements dans deux pays différents induit la prise en compte de facteurs institutionnels.

\section{Plan}

La deuxième section discute des fondements de la différenciation des collaborations à partir des deux modèles de la littérature qui ont déjà servi de référence, celui de $\mathrm{S}$. J. Kline et $\mathrm{N}$. Rosenberg qui pose la variété des formes de recherche liées à l'innovation, et celui de W. M. Cohen et D. A. Levinthal qui introduit la question de l'assimilation des connaissances. Une illustration statistique de la différence quantitative entre les relations interentreprises et les coopérations entre les entreprises et la recherche publique est présentée à partir des données sur la sous-traitance de la R-D en France.

La troisième section traite de l'évolution des processus d'innovation et de recherche au sein des établissements interrogés et présente une classification de leurs diverses sources de connaissances externes (intra-groupe et extra-groupe), ainsi qu'une analyse de la nature des divers liens de collaboration.

La quatrième section expose les caractéristiques de la demande de connaissances des unités de production classées selon leur type d'innovation. La place particulière de la recherche universitaire est traitée en relation avec les hypothèses initiales. L'impact des politiques gouvernementales et européennes d'incitation à la coopération est aussi envisagé.

Les conclusions générales sont présentées à la cinquième section.

\section{De la différenciation des connaissances nécessaires au sein des processus d'innovation à la différenciation des collaborations}

L'élaboration de notre schéma de la demande de collaborations scientifiques et technologiques suscitée par les activités innovatrices ${ }^{26}$ de la firme comprend deux étapes:

26. Schumpeter, à qui on attribue la paternité du terme d'innovation, en a distingué cinq types: (i) introduction d'un nouveau produit, (ii) introduction d'une nouvelle méthode 
122 L'économie du savoir: une économie de la collaboration?

- l'identification de besoins de connaissances et de recherches différenciés selon la phase considérée dans la chaîne d'innovation, à partir d'une relecture de l'article de 1986 de S. J. Kline et N. Rosenberg;

- l'analyse des modalités d'acquisition des différents types de connaissances nécessaires, et de l'arbitrage entre l'exécution de la R-D en interne et le recours à la recherche contractuelle, en prenant comme point de départ, le concept de capacité d'assimilation développé par W. M. Cohen et D. A. Levinthal.

Cette approche organisationnelle de l'innovation et des relations de coopération de recherche devra tenir compte du contexte institutionnel quand elle sera confrontée aux résultats des entrevues en entreprises. La demande réelle de coopération scientifique et technologique est dépendante des caractéristiques de l'offre de connaissances externes. Les «infrastructures de connaissance», les différences institutionnelles, les évolutions des systèmes sont autant d'ensembles de facteurs qui influencent les processus d'innovation et de coopération ${ }^{27}$. Les firmes interagissent avec leur

de production, (iii) ouverture d'un nouveau marché, (iv) conquête d'une nouvelle source d'approvisionnement en matières premières ou en produits semi-manufacturés, (v) réorganisation d'une industrie, les deux premiers constituant les innovations technologiques à proprement parler (cité par le Manuel d'Oslo, OCDE, Principes directeurs pour le recueil et l'interprétation des données sur l'innovation technologique - Manuel d'Oslo, Paris, 1992, p. 29-30). Depuis, la notion a encore été étendue avec la distinction entre l'innovation technologique et l'innovation organisationnelle ou encore l'innovation industrielle et l'innovation dans les services. Nous nous limitons à l'innovation entendue comme changement technologique (innovation de produits et innovation de procédés).

27. Les facteurs susceptibles d'être retenus sont nombreux et varient selon l'approche retenue: la notion de «knowledge infrastructure» de K. Smith, s'étend, au-delà des universités et des laboratoires de recherche publics, aux systèmes de formation et aux organismes de gestion des normes industrielles et de la propriété intellectuelle; voir K. Smith, «Economic infrastructure ...», op. cit. L'Économie institutionnelle considère pour sa part les institutions comme des règles du jeu, légales et comportementales, le cadre institutionnel définissant ainsi les positions et les fonctions sociales des individus et des groupes et contraignant leurs actions; voir D. C. North, Institutions, Institutional Change and Economic Performance, Cambridge, New York, Cambridge University Press, 1990. Pour B. Coriat et O. Weinstein, les institutions analysées comme contraintes et occasions pour l'innovation sont, principalement, les rapports entre les actionnaires et les dirigeants, les mécanismes de «corporate governance), les dispositifs de financement de l'innovation, les règles du droit de la propriété intellectuelle, les conventions collectives et, d'une façon générale, les relations entre le capital et le travail; voir B. Coriat et O.Weinstein, «Organizations, firms and 
environnement, et l'évolution de leurs régimes d'innovation est soumise à d'autres stratégies que celles centrées sur la R-D. Les «contraintes de sentier» et le poids des «apprentissages antérieurs» ${ }^{28}$ modèlent les structures d'innovation des entreprises ainsi que l'histoire des sites et de leurs changements de propriétaires. Le poids des fusions-acquisitions relativise la place des objectifs technologiques ${ }^{29}$.

La démarche est limitée; elle doit seulement servir de support à l'objectif qui consiste à situer la place de la recherche universitaire parmi les coopérations scientifiques et technologiques des entreprises. Il s'agit d'une approche de type qualitatif qui s'intéresse au «comment» des relations entre les régimes d'innovation des entreprises et les ressources extérieures de connaissances. La question des coûts qui résultent des relations contractuelles, telle qu'elle est envisagée, par exemple par l'économie des «coûts de transaction» et le courant néo-institutionnaliste, n'est pas abordée ici ${ }^{30}$.

\section{Variété de la recherche dans le modèle d'innovation de Kline et Rosenberg}

Alors que l'application massive de la science à la production est présentée cornme un trait central de l'économie contemporaine, de nombreux auteurs soulignent que la science n'est qu'un élément du processus d'innovation et pas nécessairement le plus important. La conception générale des rapports entre la recherche et le changement technologique présentée dans l'article de 1986 de S. J. Kline et N. Rosenberg est encore largement admise. Le modèle de liaison en chaîne (ou de la chaîne interconnectée), largement diffusé, montre les relations et les rétroactions entre les différentes phases de l'innovation au sein de l'entreprise (conception, tests, production, mise sur le marché) ainsi que les liaisons qui peuvent intervenir avec la recherche à chacune de ces étapes. Les auteurs

institutions in the generation of innovation», Research Policy, vol. 31, 2002, p. 273290.

28. G. Dosi, D. J. Teece et S. Winter, «Les frontières des entreprises: vers une théorie de la cohérence de la grande entreprise», Revue d'économie industrielle, $\mathrm{n}^{\circ}$ 51, 1990, p. 238-255.

29. B. Madeuf, G. Lefebvre et A. Savoy, Les activités de recherche en France des sociétés étrangères, Rapport pour la Direction générale de la recherche et de la technologie, MRE, Paris, 1993.

30. Il est à noter qu'un certain nombre de travaux qui se situent dans cette perspective concluent que l'internalisation de la R-D est en général plus avantageuse. 
opposent leur modèle au modèle dit linéaire, qui conçoit le processus d'innovation comme allant de la recherche à la mise sur le marché du produit, en passant par les étapes du développement et de la fabrication. Le modèle de Kline et Rosenberg est même parfois présenté comme incompatible avec l'idée selon laquelle la conception d'un produit peut être une application de la science ${ }^{31}$. En fait, les auteurs tiennent compte des deux schémas qui continuent à coexister dans la réalité: d'une part, une production scientifique qui se place en amont des processus de production industrielle et, d'autre part, des recherches suscitées au sein des différentes fonctions de la chaîne d'innovation, de la conception à la commercialisation. Un des aspects intéressants de la démarche de S. J. Kline et N. Rosenberg est, précisément, qu'ils envisagent des relations avec la science à chaque étape du processus d'innovation, ce qui les amène à discuter du type de science nécessaire:

It is also important to note that the type of science that is typically needed is different at various stages in the central-chain-of-innovation. The science needed at the first stage (design or invention) is often pure, long-range science that is indistinguishable from pure academic science in the relevant discipline. The research generated in the development stage is more often of a system nature and concerns analysis of how the components of the system interact and of the «holistic» or system properties that are generated when the components of the product envisaged are hooked together to obtain the complete function needed. [...] The research that is spawned in the production stage is more often process research: studies of how particular components can be manufactured and how the cost of manufacture can be reduced by improved special machinery or processes or by use of improved or less expensive materials ${ }^{32}$.

31. Voir par exemple le Manuel d'Oslo, op. cit., p. 20. Dans le modèle de liaison en chaîne, la recherche n'est pas considérée comme une source d'idées novatrices, mais comme un moyen de résoudre les problèmes, auquel on peut recourir à n'importe quel stade.

32. S. J. Kline et N. Rosenberg, «An Overview of Innovation», op. cit., p. 291-292. 
Les auteurs, comme dans le modèle linéaire, séparent les différentes fonctions du procès d'innovation, y compris la fonction de recherche, qu'ils segmentent. Chaque phase de la chaîne d'innovation est associée à un type de recherche particulier, dominant: la recherche à long terme avec la conception, la recherche sur les systèmes avec le développement, la recherche sur les procédés avec la production. S. J. Kline et N. Rosenberg ajoutent que les questions qui surgissent au cours du procès d'innovation sont d'abord adressées au stock de connaissances disponibles et éventuellement, en cas de carence, suscitent la recherche de connaissances nouvelles. Les auteurs distinguent deux composantes de la science: (i) la totalité du stock actuel de connaissances sur la nature, (ii) les processus par lesquels sont corrigés et augmentés ces connaissances. Ce n'est pas seulement avec la science du jour que l'on innove mais avec la somme des connaissances accumulées, et la mise en œuvre de recherches nouvelles ne se fait qu'en dernier recours, lorsque toutes les autres solutions ont été épuisées. Le recours à de nouvelles recherches, après avoir utilisé les connaissances existantes, est valable à l'intérieur de la firme comme à l'extérieur: l'entreprise exécute de la R-D ou bien a recours à la recherche contractuelle après avoir épuisé les possibilités offertes par ses savoirs et ses savoir-faire accumulés et après avoir vérifié que les connaissances n'étaient pas disponibles à l'extérieur. Mais lorsque l'option de la recherche est rendue nécessaire, le choix entre la R-D interne et la R-D externe n'est pas explicitement évoqué. La partie du chapitre de S. J. Kline et $\mathrm{N}$. Rosenberg qui traite de l'incertitude dans l'innovation signale seulement l'intérêt du maintien d'une activité scientifique au sein des entreprises, non seulement pour la production de résultats utiles à long terme, mais surtout pour sa fonction d'assimilation des avancées scientifiques dans le monde, susceptibles d'être utilisées pour des projets internes de développement. On peut cependant tirer quelques implications sur la recherche externe, de la différenciation des besoins de recherche tout au long de la chaîne innovation. S'il est clair qu'en dehors de l'entreprise elle-même, lorsqu'elle possède d'importants laboratoires de recherche, c'est la recherche universitaire qui est la mieux placée pour répondre à l'éventuelle demande de recherche à long terme pour la conception d'un nouveau produit, la réponse est plus complexe en ce qui concerne la recherche sur les systèmes, sur les procédés et d'une façon générale la recherche de solutions aux problèmes posés au cours des phases de développement et de production. Les collaborations de recherche entre les entreprises suscitées par la gestion de l'interaction des composants d'un 
système sont directement liées à la configuration de la réalisation du système, quand elle implique plusieurs entreprises. Les relations clientfournisseur entre «systèmier» ou «ensemblier» et «composantier» ont souvent une composante recherche. En revanche, l'innovation sur les procédés est difficile à protéger avec les brevets. En effet, contrairement aux produits qui circulent, les procédés restent à l'intérieur des entreprises qui les mettent en œuvre et, en conséquence, ils ne sont pas directement contrôlables en cas d'imitation. La seule protection véritablement efficace serait donc le secret, ce qui a priori exclut la collaboration. Mais les procédés industriels peuvent être proches des dispositifs expérimentaux de laboratoire, d'où la recherche de l'expertise universitaire pour l'amélioration des procédés dans certains domaines.

Si le procès d'innovation selon $\mathrm{S}$. J. Kline et $\mathrm{N}$. Rosenberg structure à la fois l'organisation de la recherche interne et la demande de connaissances externes, en revanche la fonction d'assimilation des connaissances externes par la recherche interne est seulement posée comme principe général sans être envisagée aux différentes phases de la chaîne d'innovation. Cette question sera examinée à la section suivante, mais avant, il nous faut ajouter un bref commentaire sur le point de vue des auteurs selon lequel la conception est au centre de l'innovation.

L'innovation technologique met en cuvre des activités distinctes de la recherche: conception et ingénierie, commercialisation. Le processus central de l'innovation n'est pas la science mais la conception: au début du processus, l'élément décisif est le choix du projet qui a le plus de chance de succès et ensuite, l'important est d'améliorer les procédés et de réduire les coûts. Si ce modèle d'innovation met en avant les interactions entre des unités fonctionnelles à l'intérieur et à l'extérieur de l'entreprise, il maintient la séparation des fonctions de conception, de recherche, de tests et d'essais, de production ou de marketing. Certains auteurs ont proposé d'élargir le concept de R-D pour tenir compte de la diversité des activités scientifiques et techniques qui sont mobilisées dans les processus d'innovation. Les termes de RD\&D (en anglais, research, design and development) ou R-D-E (recherche, développement, engineering), par exemple, sont parfois employés ${ }^{33}$. Mais, comme le souligne J. Perrin ${ }^{34}$, le sigle R-D-E ne représente pas une simple extension du concept de R-D: en

33. Dans les entreprises françaises, on rencontre des intitulés de département ou de fonction tels que R-D-I (recherche, développement et industrialisation) ou R-D-E (recherche, développement et études).

34. J. Perrin, Concevoir l'innovation industrielle, Paris, CNRS Éditions, 2001, p. 125. 
effet le développement de produits (ou de procédés) auquel il est fait référence dans le nouveau concept de R-D-E diffère de la définition du développement expérimental par $1^{\prime} O C D E^{35}$. Dans la conception traditionnelle, le développement expérimental représente la phase qui, dans le prolongement d'une recherche appliquée, va permettre d'aboutir à un premier prototype. En revanche, le développement, selon la nouvelle terminologie, peut désigner l'ensemble des activités qui participent à la conception du produit (du procédé), ou à sa reconception dans un environnement changeant. Sont incluses, en particulier, les activités de conception des bureaux d'études et les activités de contrôle des laboratoires d'essais. Mais, l'idée d'une fusion de la conception et du développement introduit une nouvelle séparation, à un autre point de la chaîne d'innovation, entre le développement et la production. De façon concrète, des entreprises, ayant réuni les fonctions d'études et d'industrialisation dans un pôle développement, ont constaté que la distance entre industrialisation et fabrication s'était accentuée. On doit aussi admettre que le poids des différentes fonctions et leurs relations ne sont pas nécessairement les mêmes selon l'industrie considérée. D'un point de vue opératoire, la R$\mathrm{D}$ reste sans doute la fonction la plus commode à évaluer, même si son contenu n'est pas homogène ${ }^{36}$.

\section{Capacité d'absorption de la firme, diversification de l'innovation et coopération}

\section{Le concept de capacité d'absorption et ses limites}

Les échanges technologiques ne sont pas des phénomènes instantanés, mais demandent des apprentissages. Les difficultés inhérentes aux transferts technologiques ont suscité une littérature sur les formes d'apprentissage technologique (par la pratique, par l'usage, etc.), mais au-delà de cet aspect descriptif, la question qui nous intéresse porte sur les déterminants de la capacité d'apprentissage technologique. Une première

35. OCDE, La inesure des activités scientifiques et technologiques - Manuel de Frascati, Paris, 1993, p. 31.

36. C'est le point de vue exprimé par l'OCDE dans le Manuel d'Oslo, op. cit., p. 26. Comme point de départ pour l'analyse de l'innovation, on pourrait prendre la R-D qui revêt une grande variété de formes fonctionnelles liées à la solution des problèmes. Même si la plupart des données mises à notre disposition à l'heure actuelle ont trait à la R-D, les activités réelles des services de R-D sont en fait mal connues. 
perspective a été ouverte par Cohen et Levinthal ${ }^{37}$ pour le traitement des conditions nécessaires à l'assimilation des connaissances externes par l'entreprise. La R-D de la firme génère de l'innovation, mais elle représente également une capacité d'absorption (d'apprentissage) des connaissances externes, qu'il s'agisse d'innovations de produit ou de procédé, ou encore de recherches de base. Les auteurs établissent une relation quantitative positive entre le taux d'assimilation des connaissances externes et le volume des dépenses de R-D de l'entreprise. La question des modalités d'assimilation des connaissances internes n'est pas envisagée. Cependant, des travaux économétriques récents remettent en cause l'effet positif de la capacité d'absorption sur l'incitation de la firme à investir dans la R-D, en montrant qu'il y a dé-incitation à investir dans la R-D à partir d'une certaine taille du marchée ${ }^{38}$. La relation quantitative simple, établie par Cohen et Levinthal, a été affinée par A. Arora et A. Gambardela $^{39}$, qui postulent que la capacité scientifique interne de la firme permet d'évaluer les connaissances externes et que sa capacité technologique interne permet d'exploiter les plus rentables. La capacité scientifique est reliée au nombre de publications des chercheurs de la firme et la capacité technologique aux dépenses de R-D. La délimitation plus stricte de la capacité d'assimilation, envisagée comme une recherche d'informations scientifiques basée sur une implication des chercheurs industriels dans la production universitaire, semble plus réaliste que l'absorption conçue comme une relation globale entre connaissances internes et connaissances externes, mais alors qu'une distinction est faite entre capacité scientifique et capacité technologique d'assimilation, il n'y a pas de différenciation a priori des informations recherchées.

Une autre façon de résoudre la question de l'assimilation des connaissances externes consiste à considérer que la coopération est un moyen privilégié d'acquisition de connaissances externes, autrement dit de considérer comme résolu le problème de l'absorption, à partir du moment où il $\mathrm{y}$ a collaboration scientifique et technologique. Ainsi, S. Lhuillery définit des moyens externes d'assimilation de la technologie, représentés par les relations avec d'autres organismes publics ou privés, à côté des

37. W. M. Cohen et D. A. Levinthal, «Innovation and Learning ...», op. cit.

38. L. A. Grünfeld, «Meet me halfway but don't rush: absorptive capacity and strategic RD investment revisited», International Journal of Industrial Organization, vol. 21, 2003, p. 1091-1109.

39. A. Arora et A. Gambardella, «Evaluating technological information and utilising it Scientific knowledge, technical capability, and external linkages in biotechnology», Journal of Economic Behavior and Organization, vol. 24, n 1, 1994, p. 91-114. 
moyens internes d'assimilation des connaissances externes, que constitue l'activité interne de R-D, mise en avant par Cohen et Levinthal:

Les accords de R-D, formels ou informels, peuvent être considérés comme des moyens, à part entière, d'absorption mis en œuvre par les firmes ${ }^{40}$.

Des auteurs soulignent, par ailleurs, que les grands groupes, comme les multinationales de la pharmacie, en tant qu'organisateurs à leur profit de réseaux de compétences, ont des objectifs d'utilisation des connaissances produites par leurs partenaires, sans chercher nécessairement à assimiler cette connaissance ${ }^{41}$.

Caractère risqué de la recherche versus caractéristiques systémiques de l'innovation

Les rapports entre la recherche interne et la recherche externe peuvent être présentés dans une autre perspective: d'une part, l'intégration de la R$\mathrm{D}$ est nécessaire pour qu'elle joue son rôle d'apprentissage collectif visà-vis des autres fonctions internes et, d'autre part, son externalisation est voulue pour des raisons de flexibilité ${ }^{42}$. La coopération qui répartit les coûts d'irréversibilité, liés à l'internalisation, entre plusieurs organisations serait un moyen de résoudre la contradiction entre apprentissage et flexibilité. Mais cette approche repose sur la conception standard de la recherche comme investissement incertain. La variété des demandes de connaissance et de recherche à chaque étape de la chaîne d'innovation, telle qu'elle a été décrite à la section précédente, plaide en faveur d'une vision différente de la recherche, ou, plus précisément, de l'existence d'un autre type de recherche, à côté de la recherche à long terme marquée par l'incertitude. La recherche de solutions qui accompagne le procès de développement du produit et la recherche de solutions pour améliorer les procédés, sont souvent des recherches à très court terme, entièrement programmées. L'innovation technologique qui oriente cette recherche présente

40. S. Lhuillery, Recherche et développement et transferts de connaissances technologiques, Thèse de doctorat de Sciences économiques, Université Paris Nord, 1996, p. 93.

41. A. Bureth, «Nature composite ...», op. cit., p. 15.

42. D. Foray, «Repères pour une économie des organisations de recherchedéveloppement», Revue d'économie politique, vol. 101, $\mathrm{n}^{\circ} 5$, septembre - octobre, 1991. 
également des caractéristiques systémiques de plus en plus marquées. Les caractéristiques architecturales de l'innovation façonnent les alliances interentreprises de conception et de développement.

Un schéma diversifié des processus d'innovation, de recherche et de coopération.

On peut schématiser, de la façon suivante, les deux types de recherche évoqués et les formes de collaborations qui leur sont associées:

(i) La recherche à plus ou moins long terme pour préparer de nouvelles innovations nécessite généralement l'acquisition de connaissances externes. La complexité des connaissances à acquérir implique une capacité d'évaluation des connaissances externes disponibles, mais l'objectif principal est l'utilisation de ces connaissances, et non pas leur assimilation. La coopération est un moyen de partager les risques de la recherche à long terme, mais les accords sont aussi des formes d'appropriation des connaissances. Le but des groupes industriels est l'appropriation des résultats qui sont apportés par les laboratoires universitaires (et parfois des entreprises spécialisées). Des stratégies se développent qui visent à étendre et à contrôler le domaine d'appropriation de la recherche (par exemple sous la forme de consortium).

(ii) La recherche comme activité de soutien à l'innovation, encadrée par les exigences du procès d'innovation, suscite des alliances fondées sur l'architecture de l'innovation. La forme élémentaire de coopération due au caractère systémique de l'innovation technologique est la relation client/fournisseur. La conception modulaire des produits et systèmes amplifie les coopérations interentreprises et diversifie leurs configurations. Les alliances nouées sur cette base ont plus de chance d'être passées au niveau de l'entreprise, de la division ou de l'établissement, qu'au niveau du groupe. S'ill y a intervention de la recherche universitaire, c'est comme prestataire de service ou pour apporter une expertise.

La distinction qui est faite entre les deux types de recherche et de coopération est analytique; elle vise à donner des repères pour l'étude des relations concrètes des entreprises de notre enquête. Les différentes formes de recherche et de coopération peuvent se combiner. La question du lien 
entre la nature des recherches et des coopérations, et les formes d'organisation de la firme, doit aussi être examinée à partir des enquêtes.

\section{Un résultat statistique: les entreprises, source majeure de technologie pour les entreprises}

Le tableau 1 donne les flux de R-D sous-traitée par les entreprises en France (recensement à partir d'une enquête annuelle de toutes les entreprises qui déclarent des activités de R-D). La sous-traitance ne représente qu'une partie de la coopération technologique qui peut prendre d'autres formes contractuelles ou marchandes, unilatérales ou bilatérales (cession et échange de licences, dépôt de marque en commun, accords de partage de technologie, participation à consortium...) et inclut également des relations informelles et des relations interpersonnelles. Il est cependant raisonnable de penser que la seule sous-traitance constitue une bonne représentation de l'ensemble des modalités de coopération, d'une façon générale, et plus particulièrement en ce qui concerne la large prédominance des flux interentreprises sur les flux en direction des universités et des laboratoires publics. En effet, d'une part, l'hypothèse d'une certaine complémentarité entre relations formelles et relations informelles n'est pas invraisemblable, d'autre part, les formes contractuelles autres que la soustraitance semblent plus impliquer les relations interentreprises que les échanges entre les entreprises et la recherche publique. 
132 L'économie du savoir: une économie de la collaboration?

\section{Tableau 1}

\section{Évolution de la structure d'exécution de la R-D sous-traitée par les entreprises (France, 1994-2000 en \%)}

\begin{tabular}{|c|c|c|c|c|c|c|c|}
\hline $\begin{array}{l}\text { LA SOUS- } \\
\text { TRAITANCE DE R-D } \\
\text { DES ENTREPRISES } \\
\text { PAR SECTEUR } \\
\text { D'EXÉCUTION } \\
\text { DE } 1994 \text { Ȧ 2000 } \\
\text { (STRUCTURE) } \\
\end{array}$ & 1994 & 1995 & 1996 & 1997 & 1998 & 1999 & 2000 \\
\hline \multicolumn{8}{|l|}{ Dépenses exécutées par } \\
\hline Secteur de l'État & 5,4 & 5,5 & 6,1 & 6,0 & 5,8 & 5,6 & 4,7 \\
\hline CEA & 3,7 & 3,8 & 4,3 & 4,3 & 4,4 & 4,1 & 3,4 \\
\hline Autres organismes & 1,6 & 1,7 & 1,7 & 1,8 & 1,5 & 1,5 & 1,3 \\
\hline $\begin{array}{l}\text { Enseignement } \\
\text { supérieur }\end{array}$ & 2,6 & 2,8 & 2,6 & 2,7 & 2,8 & 2,1 & 2,0 \\
\hline $\begin{array}{l}\text { Universités et établis- } \\
\text { ements d'enseignement } \\
\text { supérieur }\end{array}$ & 2,1 & 2,3 & 2,1 & 2,2 & 2,3 & 1,7 & 1,6 \\
\hline CNRS & 0,5 & 0,5 & 0,5 & 0,5 & 0,5 & 0,5 & 0,4 \\
\hline $\begin{array}{l}\text { ISBL (Institutions sans } \\
\text { buts lucratifs) }\end{array}$ & 0,9 & 0,8 & 0,8 & 0,9 & 0,9 & 0,4 & 1,0 \\
\hline Entreprises en France & 68,2 & 68,4 & 67,0 & 68,6 & 67,4 & 71,8 & 68,0 \\
\hline Filiales de groupes & 29,4 & 27,5 & 26,9 & 26,7 & 20,1 & 19,6 & 14,2 \\
\hline Autres entreprises & 37,8 & 39,9 & 39,1 & 40,7 & 45,7 & 51,2 & 53,4 \\
\hline $\begin{array}{l}\text { Organismes profes- } \\
\text { ionnels }\end{array}$ & 1,1 & 1,1 & 1,0 & 1,2 & 1,6 & 1,0 & 0,4 \\
\hline $\begin{array}{l}\text { Étranger et } \\
\text { organismes } \\
\text { internationaux }\end{array}$ & 22,9 & 22,4 & 23,5 & 21,8 & 23,1 & 20,1 & 24,3 \\
\hline Filiales de groupes & 6,8 & 7,2 & 7,8 & 7,7 & 7,6 & 7,4 & 9,5 \\
\hline Autres entreprises & 14,4 & 13,7 & 14,6 & 13,2 & 14,2 & 11,7 & 12,5 \\
\hline $\begin{array}{l}\text { Organismes interna- } \\
\text { ionaux ou étrangers }\end{array}$ & 1,7 & 1,5 & 1,1 & 0,9 & 1,3 & 1,0 & 2,3 \\
\hline TOTAL & 100 & 100 & 100 & 100 & 100 & 100 & 100 \\
\hline $\begin{array}{l}\text { Total dépenses } \\
\text { extérieures de R-D } \\
\text { (millions d'euros) }\end{array}$ & 4162 & 4151 & 4001 & 3993 & 4107 & 4671 & 5068 \\
\hline
\end{tabular}

Sources: calculs effectués à partir des données MEN/DPD C3 
Non seulement les secteurs de l'État et de l'Enseignement supérieur n'exécutent qu'une très faible part de la R-D sous-traitée par les entreprises, mais, de plus, cette part est en régression de telle sorte que les deux secteurs réunis exécutent en 2000 moins de $7 \%$ de la dépense extérieure de R-D des entreprises. Encore faut-il tenir compte de ce que le Commissariat à l'énergie atomique (CEA), à lui seul, est destinataire de la moitié des contrats passés par les entreprises avec le secteur public (État et Enseignement supérieur), ce qui inclut les contributions versées au CEA par ses filiales industrielles. Étant donné que la $\mathrm{R}-\mathrm{D}$ sous-traitée représente à peine le quart des dépenses totales de R-D des entreprises, les universités et les laboratoires publics exécutent moins de $2 \%$ de la recherche des entreprises, et le CNRS et les universités, à eux seuls, $0,5 \%$. Non seulement, on est loin de l'hybridation, parfois annoncée, entre la science et l'industrie, mais on semble même s'en éloigner. En ce qui concerne l'ensemble des dépenses extérieures de R-D des entreprises, les résultats fournis par les mêmes sources de la Direction de la programmation et de la recherche du ministère de l'Éducation nationale (DEP MEN) montrent, de façon également surprenante, que leur part dans le total des dépenses de R-D des entreprises est très stable: $23 \%$ en 1994 contre $24 \%$ en 2000 , avec entre les deux une diminution puis un redressement de la part de la sous-traitance.

Les flux interentreprises, qui représentent plus de $92 \%$ de la R-D soustraitée, évoluent de façon très différenciée, selon que l'on observe les contrats passés à l'intérieur du groupe ou avec les autres entreprises hors du groupe, ou bien qu'il s'agisse de la sous-traitance en France ou entre la France et l'étranger. La délocalisation de la R-D ne suffit pas à expliquer l'augmentation des flux avec les filiales étrangères et la diminution des flux intra-groupes en France, car cette dernière a été beaucoup plus brutale que la première, sa part étant divisée par deux sur la période. L'ampleur du transfert de la sous-traitance de R-D de l'intérieur des groupes vers l'extérieur, en France, témoigne de la profondeur des changements non seulement de l'organisation de la recherche des grands groupes mais aussi de leurs stratégies globales. Parmi les facteurs susceptibles d'expliquer ce qui constitue une externalisation de la R-D hors du groupe, on peut évoquer le contrôle (ou la création) de petites entreprises de haute technologie en lieu et place du développement des capacités internes de recherche des groupes (en particulier dans l'électronique et les biotechnologies). L'évolution de la répartition sectorielle des activités de R-D peut aussi jouer un rôle avec l'accentuation du poids de l'industrie 
134 L'économie du savoir: une économie de la collaboration?

automobile qui sous-traite peu au sein des groupes mais multiplie les partenariats intergroupes.

\section{Les unités de production visitées: présentation analytique des formes de l'innovation et des sources de connaissances externes}

\section{Présentation des établissements}

Les résultats qui figurent au tableau 2 ont été collectés au sein des établissements où sont élaborés les composants, équipements et systèmes optoélectroniques. Certains de ces établissements groupent également d'autres activités connexes, de microélectronique ou d'infrastructure radio par exemple. En règle générale, l'essentiel de la fabrication d'un ou plusieurs produits ou systèmes optiques et optoélectroniques est concentrée sur un seul site, mais il existe des exceptions: (i) la répartition d'une division sur plusieurs sites résulte d'une politique permanente d'acquisitions et de ventes de la maison mère, (ii) deux petits sites travaillent en étroite collaboration.

La définition des frontières des unités que nous avons retenues correspond à ce que 1'OCDE nornme «unité du type entreprise ${ }^{43}$ », c'està-dire des unités qui possèdent un certain degré d'indépendance économique, et qui, lorsque l'entreprise travaille dans plusieurs branches d'activité, peuvent être une unité plus petite que l'entreprise, un établissement ou une division spécialisée, par exemple.

Seuls les entreprises, établissements ou divisions dont l'activité principale est une activité de production ont été retenus. Les centres de R$\mathrm{D}$, les start-up encore au stade de développement du produit avant sa première mise sur le marché, ainsi que les entreprises qui se sont spécialisées dans une activité de services d'études n'ont pas été pris en considération dans le cadre de la présente exploitation.

L'échantillon ainsi constitué de 17 unités n'a évidemment pas de visées statistiques, eu égard à sa taille. Il est susceptible cependant de donner lieu à certaines classifications. Il s'agit d'une collection de cas particuliers qui présentent une certaine homogénéité fonctionnelle (production de produits nouveaux) et une variété a priori, en ce qui concerne la taille, la localisation, la propriété du capital et les marchés des produits. Pour des raisons de confidentialité demandée par certaines des entreprises interrogées, l'identité des établissements visités n'est pas donnée.

43. OCDE, Manuel d'Oslo, op. cit., p. 62. 
Résultats d'enquêtes dans les secteurs des technologies optoélectroniques 135

Tableau 2

Présentation des unités de production et de leurs collaborations

\begin{tabular}{|c|c|c|c|c|c|c|c|c|c|c|c|c|c|c|}
\hline & 窎 & 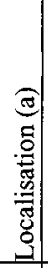 & 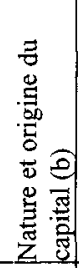 & 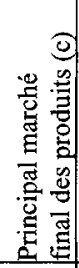 & 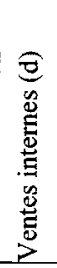 & 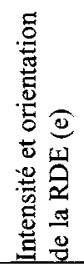 & 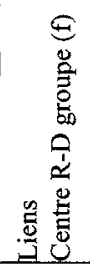 & 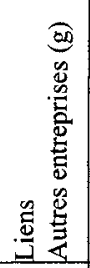 & 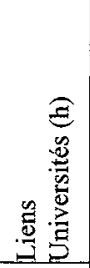 & 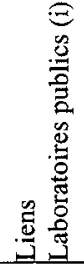 & 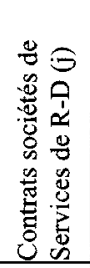 & 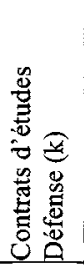 & 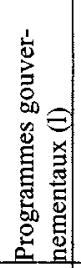 & 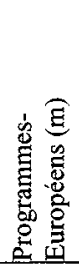 \\
\hline P1 & 1600 & A & $\begin{array}{l}\mathrm{G} \\
\mathrm{NA}\end{array}$ & $\mathrm{T}$ & 1 & $\begin{array}{l}= \\
\mathrm{c}\end{array}$ & 2 & 0 & 1 & 0 & 0 & 0 & 0 & 0 \\
\hline $\mathrm{P} 2$ & 1180 & $E$ & $\begin{array}{l}\mathrm{G} \\
\mathrm{B}\end{array}$ & M & 0 & + & 2 & 2 & 2 & 0 & 0 & 1 & 0 & 0 \\
\hline P3 & 1150 & $E$ & $\begin{array}{l}\mathrm{G} \\
\mathrm{NA}\end{array}$ & $\mathrm{A}$ & 0 & - & 1 & 1 & 1 & 0 & 0 & 0 & 0 & 0 \\
\hline $\mathrm{P} 4$ & 750 & A & $\begin{array}{l}\mathrm{G} \\
\mathrm{NA}\end{array}$ & $\mathrm{T}$ & 0 & - & 1 & 0 & 2 & 1 & 0 & 0 & 0 & 0 \\
\hline P5 & 587 & $\bar{E}$ & $\begin{array}{l}\mathrm{G} \\
\mathrm{B} / \mathrm{F}\end{array}$ & $\overline{\mathrm{M}}$ & 0 & $=$ & 2 & 2 & 2 & 0 & 0 & 1 & 0 & 0 \\
\hline $\mathrm{P} 6$ & 439 & $F$ & $\begin{array}{l}\mathrm{G} \\
\mathrm{F}\end{array}$ & $\mathrm{M} / \mathrm{A}$ & 0 & $=$ & 2 & 2 & 2 & 2 & 0 & 1 & 0 & 0 \\
\hline P7 & 320 & $\mathrm{~F}$ & $\begin{array}{l}G \\
F\end{array}$ & $T$ & 1 & $\begin{array}{l}+ \\
c\end{array}$ & 2 & 0 & 1 & 2 & 0 & 0 & 0 & 0 \\
\hline P8 & 250 & $F$ & $\begin{array}{l}\mathrm{G} \\
\mathrm{J}\end{array}$ & $\mathrm{T}$ & 1 & - & 2 & 1 & 0 & 2 & 0 & 0 & 0 & 0 \\
\hline P9 & 200 & $E$ & $\begin{array}{l}G \\
B\end{array}$ & $\mathrm{M} / \mathrm{T}$ & 0 & $\begin{array}{l}= \\
c\end{array}$ & 2 & 1 & 2 & 0 & 0 & 1 & 0 & 0 \\
\hline $\mathrm{P} 10$ & 200 & $\mathrm{~F}$ & $\begin{array}{l}\mathrm{M} \\
\mathrm{F}\end{array}$ & $\mathrm{T} / \mathrm{m}$ & 0 & $=$ & 0 & 2 & 1 & 2 & 0 & 0 & 0 & 0 \\
\hline P11 & 200 & $\dot{\mathrm{F}}$ & $\begin{array}{l}M \\
F\end{array}$ & $\mathrm{M}$ & $\overline{0}$ & $\bar{c}=$ & 2 & 1 & 2 & 2 & 0 & 1 & 1 & 0 \\
\hline $\mathrm{P} 12$ & 200 & $\mathrm{~F}$ & $\begin{array}{l}P \\
F\end{array}$ & $\mathrm{~T} / \mathrm{m}$ & 0 & $=$ & 0 & 2 & 2 & 2 & 0 & 1 & 1 & 1 \\
\hline P13 & 120 & A & $\begin{array}{l}\mathrm{G} \\
\mathrm{B}\end{array}$ & $\mathrm{A} / \mathrm{m}$ & 0 & $\bar{c}$ & 0 & 2 & 2 & 0 & 1 & 0 & 0 & 1 \\
\hline P14 & 104 & $\mathrm{E}$ & $\begin{array}{l}\mathrm{P} \\
\mathrm{NA}\end{array}$ & $\mathrm{A} / \mathrm{m}$ & 0 & c & 1 & 2 & 1 & 0 & 0 & I & 0 & 0 \\
\hline P15 & 42 & $\mathrm{~A}$ & $\begin{array}{l}\mathrm{M} \\
\mathrm{B}\end{array}$ & $\mathrm{T} / \mathrm{m}$ & 0 & $\begin{array}{l}\overline{\bar{c}} \\
\mathrm{c}\end{array}$ & 0 & 2 & 1 & 0 & 0 & 0 & 0 & 0 \\
\hline P16 & 35 & $\mathrm{~A}$ & $\begin{array}{l}\text { I } \\
\text { B }\end{array}$ & $\mathrm{A} / \mathrm{m}$ & 0 & $=$ & 0 & 2 & 2 & 0 & 0 & 0 & 1 & 0 \\
\hline P17 & 26 & $\mathrm{~F}$ & $\begin{array}{l}\mathrm{G} \\
\mathrm{F} \\
\end{array}$ & $\mathrm{A} / \mathrm{m}$ & 0 & $\bar{c}$ & 0 & 0 & 2 & 2 & 0 & 1 & 0 & 1 \\
\hline
\end{tabular}


136 L'économie du savoir: une économie de la collaboration?

Notes :

(a) A: Angleterre, E: Écosse, F: France

(b) G: grand groupe multinational, M: moyen groupe, P: petit groupe

l: entreprise indépendante, $N A$ : nord-américain, $B$ : britannique, $F$ : français, $I$ : italien

(c) $\mathrm{T}$ : télécommunications, $\mathrm{M}$ : équipements militaires, $\mathrm{A}$ : autres industries, $\mathrm{m}$ : équipements militaires minoritaires

(d) 0 : moins de $50 \%, 1: 50 \%$ et plus

(e) RDE: recherche, développement et études,

= intensité de RDE moyenne - dépenses ou effectif de RDE/chiffre d'affaires ou effectif total, compris entre 9 et $13 \%$,

+: intensité de RDE supérieur à $13 \%$,

$\because$ intensité de RDE inférieure à $9 \%$,

c: l'innovation de procédés est une préoccupation majeure

(f) 0: il n'y a pas de structure de groupe, ou il n'existe pas de centre de recherche au sein du groupe; ou il n'y a pas de relations avec un centre de recherche du groupe, 1: relations faibles avec un centre de recherche du groupe, 2: relations fortes avec un centre de recherche du groupe

(g) 0: l'entreprise n'a pas de partenariat technologique (à l'extérieur de son groupe), 1: la coopération est seulement liée à la spécification des produits, 2 : il y a accès à la R-D du partenaire

(h) 0: pas de contacts avec les universités (ni avec le CNRS), 1: relations de services et de consultance, et relations informelles, 2: relations contractuelles de recherche (y compris le financement de doctorants)

(i) 0: pas de contacts avec les laboratoires publics, 1: relations de services 2: relations de recherche

(j) 0: pas de relations avec des laboratoires indépendants ou des sociétés de services de R-D

1: contrats avec des laboratoires indépendants ou des sociétés de services de R-D

(k) 0: pas de contrats d'études financés par le ministère de la Défense

1: contrats d'études Défense

(1) 0: pas de R-D financée par des agences gouvernementales (hors défense)

1: participation à des projets avec financement gouvernemental (hors défense)

(m) 0: pas de participation à des programmes européens 1: participation à des programmes européens

\section{Activités de R-D et innovation au sein des unités de production}

\section{Raccourcissement des délais de développement}

La réduction des coûts de développement n'est pas un objectif propre aux fabricants de composants et systèmes optoélectroniques, mais c'est un enjeu important dans un secteur où le développement d'un produit peut dépasser dix années (pour des systèmes optroniques ${ }^{44} \mathrm{du}$ domaine

44. Originellement et par définition, l'optoélectronique est le domaine des composants optoélectroniques, alors que l'optronique rassemble les équipements et systèmes combinant de l'optique, de l'électronique, de l'électro-optique et des composants optoélectroniques. Mais, l'usage est plutôt de réserver le terme d'optronique aux systèmes des domaines militaires et spatiaux, alors que le terme d'optoélectronique a tendance à être appliqué aux composants comme aux équipements et systèmes, particulièrement dans le domaine des télécommunications optiques. 
militaire, mais aussi pour certains composants des télécommunications optiques). Le raccourcissement des délais de développement prend souvent la forme d'un développement et d'une industrialisation en parallèle. La mise au point des cycles de fabrication est une opération souvent longue, qui peut demander plus d'une année. La fabrication doit entrer le plus tôt possible dans la chaîne de développement. "L'engineering en parallèle» qui implique les équipes d'industrialisation dans les phases de conception et de développement des nouveaux produits facilite le démarrage en fabrication et l'accélération de la montée en cadence. Formaliser l'amont comme l'aval, mener de front conception et industrialisation ou tout au moins tenir compte de l'industrialisation dès la conception et commencer à résoudre les problèmes d'industrialisation dès ce stade permettent d'importants gains de temps. L'utilisation de «briques technologiques de base» en recherche et en fabrication contribue aussi aux différentes économies de temps, de conception, de transfert en fabrication et aussi de qualification des nouveaux produits.

Ces dispositions technologiques et organisationnelles ont eu des résultats. La réduction d'un facteur deux, sur une période d'environ cinq ans, de la séquence, recherche amont - développement - passage en fabrication, est avancée par plusieurs entreprises. En raison de la diversité des produits optoélectroniques, l'amplitude des temps de conception et de développement, comprise entre dix ans et six mois, reste cependant élevée ${ }^{45}$. D'autres facteurs tendent à allonger les délais entre la conception et la réalisation des produits, particulièrement la complexité croissante des tests qui peuvent occuper jusqu'à $90 \%$ du temps de développement (les $10 \%$ restants étant consacrés à l'écriture des algorithmes) ${ }^{46}$.

45. La tendance au rapprochement du développement de la production, et à la réduction des délais de développement, n'exclut pas, tout au moins au niveau des leaders mondiaux de l'optoélectronique, des recherches à très long terme: au moment de l'enquête, des recherches étaient menées dans les laboratoires centraux du premier groupe de notre échantillon, pour des produits qui devraient être mis sur le marché en 2010.

46. Dans les télécommunications optiques, cette complexification des tests est liée à la conception des micro-systèmes optiques intégrés, c'est-à-dire le regroupement sur une seule puce d'un maximum de composants hétérogènes (optiques et électroniques) et hybrides, pour simplifier les interconnexions optiques. 
138 L'économie du savoir: une économie de la collaboration?

Innovation produit: tendance à la personnalisation et compromis vers la standardisation

La demande de produits personnalisés est globalement en forte croissance. Cependant, entre les produits sur catalogue et ceux sur commande, il existe des formules intermédiaires. On peut même considérer que les produits standard et les produits personnalisés ne constituent pas véritablement deux catégories et qu'il y a un continuum entre les deux pôles, chaque produit étant un ensemble d'éléments standard et d'éléments sur commande, dont les proportions respectives varient. Il y a une tension entre la personnalisation demandée par les clients et la standardisation qui réduit les coûts de production. Cette antinomie diffère selon les produits, dont les séries peuvent, par ailleurs, varier d'une dizaine à des centaines de milliers d'unités. La conception modulaire de l'architecture des produits est un moyen de gestion de cette contradiction.

La production de plus des deux tiers des entreprises de l'échantillon est plutôt personnalisée, mais comme le précise le manuel d'Oslo ${ }^{47}$, une production personnalisée n'est pas systématiquement une innovation. Dans le domaine de l'optoélectronique, la demande porte souvent sur la miniaturisation de produits existants ${ }^{48}$, ce qui nécessite de longs développements pour les équipements militaires, mais pas pour la fibre optique ni les télécommunications optiques.

\section{Concurrence par l'innovation de procédés}

Le tableau 2 montre que, pour plus de la moitié des entreprises, les innovations de procédés sont une préoccupation majeure. Ces innovations de procédés impliquent des modifications qui portent sur l'équipement et sur l'organisation de la production.

L'explication, qui peut être donnée à l'importance prise par les innovations de procédés, a un double aspect:

(i) Le premier est d'ordre technologique. Les procédures technologiques d'assemblages du laser et de la fibre ont à résoudre le problème difficile de l'alignement du laser et de la fibre. L'utilisation du phosphure d'indium pour la fabrication des composants optoélec-

47. Op. cit., p. 33.

48. Sur un plan général, les nanostructures sont d'ailleurs la toile de fond de l'évolution de la microélectronique et de l'optoélectronique. 
troniques nécessite des dispositifs de sécurité élevés. Alors que l'assemblage dans le silicium, le matériau des composants traditionnels, est une opération de pure main-d'œuvre, l'assemblage de la fibre est au contraire une opération technique difficile nécessitant une expertise en micro-optique, micromécanique et métallurgie.

(ii) Le second aspect, lié au premier, est d'ordre économique: sur chaque type de marché, la plupart des entreprises mettent en œuvre les mêmes produits issus de la recherche de pointe et ne peuvent se départager que sur les coûts de fabrication liés à des améliorations des techniques de production. Le schéma s'applique particulièrement bien aux télécommunications optiques, où le «Front End» (l'ensemble des activités concernant la puce) dépend de la recherche amont, alors que le Back End (ensemble des activités concernant l'assemblage) est conçu et développé par chaque entreprise. Le recours à l'expertise des laboratoires universitaires et publics pour l'innovation de procédés pose des questions de confidentialité, d'autant plus que la protection par le brevet est difficile, car le procédé est alors connu sans qu'il soit possible de vérifier s'il n'est pas repris à l'intérieur d'une entreprise concurrente. Dans le domaine de l'optronique militaire, la reconversion partielle des firmes vers les marchés civils a eu pour effet de les aligner sur le modèle de concurrence par l'innovation de procédés, les avantages dus au financement des marchés d'études militaires ayant tendance à disparaître. Il y a également un autre ensemble d'activités vers lesquelles se déplace la concurrence: celles qui concernent l'aspect du produit, le «design» et le «packaging», celui-ci étant particulièrement important dans le domaine des produits et systèmes optoélectroniques.

\section{Évaluation de l'intensité de l'innovation et de la recherche des unités de production}

Les entreprises visitées ont été interrogées sur la part de leur chiffre d'affaires générée par les ventes des produits introduits au cours des trois dernières années. Seule la moitié des établissements ont donné une réponse précise à cette question. Pour certaines entreprises, le développement continu des produits rend difficile la distinction entre les nouveaux et les anciens produits. La fréquente personnalisation des produits peut aussi compliquer la définition de la nouveauté. Non seulement la proportion de produits nouveaux n'est pas toujours bien identifiable, mais, de plus, bien 
qu'il soit d'un usage courant dans les grandes enquêtes internationales, l'utilisation de ce critère pour mesurer le comportement innovant de la firme prête à discussion. Un pourcentage identique de nouveauté n'a pas la même signification, selon qu'il s'applique à des produits destinés aux marchés civils ou aux marchés militaires; les produits de ce dernier type demandent en général des développements beaucoup plus longs, parfois supérieurs à dix ans, avec pour conséquence un taux plus faible de renouvellement des produits. De plus, avec cette focalisation sur la part des produits nouveaux, on ne tient pas compte de l'innovation de procédés qui, comme nous l'avons vu, tient une place importante dans les secteurs industriels mettant en cuvre des technologies optiques. Il est donc difficile d'accorder une valeur absolue de mesure de l'innovation, aux résultats obtenus en prenant la part du chiffre d'affaires due aux produits introduits depuis trois ans. On se contentera d'observer qu'il y a une grande variété des pourcentages - de moins $10 \%$ à $100 \%$ - et qu'il n'apparaît pas de discordances flagrantes quand on croise, comme ci-dessous, ces chiffres avec l'intensité de la R-D (c'est-à-dire le rapport entre les dépenses de R-D et le chiffre d'affaires).

\begin{tabular}{|l|c|c|c|c|}
\hline \multicolumn{2}{|c|}{} & \multicolumn{3}{|c|}{ Intensité de la $R-D$} \\
\cline { 3 - 5 } \multicolumn{2}{|c|}{} & $<9 \%(-)$ & $9-13 \%(=)$ & $>13 \%(+)$ \\
\hline $\begin{array}{l}\text { Part du chiffre } \\
\text { d'affaires due aux } \\
\text { produits introduits } \\
\text { depuis 3 ans }\end{array}$ & $90 \grave{a} 100 \%$ & & $\mathrm{P} 1$ & $\mathrm{P} 7$ \\
\cline { 2 - 5 } & $40 \grave{a} 60 \%$ & & $\mathrm{P} 9, \mathrm{P} 16$ & $\mathrm{P} 2$ \\
\cline { 2 - 5 } & $0 \grave{a} 20 \%$ & $\mathrm{P} 14$ & $\mathrm{P} 10, \mathrm{P} 15$ & \\
\hline
\end{tabular}

Les entreprises ayant une faible intensité de R-D $(<9 \%)$, qui n'ont pas donné d'informations chiffrées sur la part des produits nouveaux, semblent appartenir au groupe à faible niveau d'intensité d'innovation de produits, si l'on se base sur des réponses qualitatives apportées à cette question. Ainsi dans de nombreux domaines, l'entreprise P3 est en position d'imitatrice, se contentant d'introduire des modifications mineures dans les produits. S'orientant vers une production élargie, P4 concentre ses efforts sur l'accroissement de la vitesse de production et la réduction des coûts, tandis que P13 vise surtout l'amélioration du rendement des procédés de production. C'est le centre de recherche du groupe qui met au point les produits nouveaux fabriqués par P8, et pour P17, l'adaptation systématique du 
produit à chaque client ne peut être prise en compte comme une réelle activité d'innovation.

La définition des classes d'intensité de R-D utilisée (niveau faible, inférieur à $9 \%$; niveau moyen, compris entre 9 et $13 \%$; niveau élevé, supérieur à $13 \%$ ) est propre à notre échantillon d'entreprises. Si on avait eu recours à la classification recommandée par l'OCDE (niveau faible, inférieur à $1 \%$; niveau moyen, compris entre 1 et $4 \%$; niveau élevé, supérieur à $4 \%)^{49}$, toutes les entreprises étudiées seraient considérées comme ayant une intensité de R-D élevée. Bien que nous n'ayons retenu que les unités de production, et pas les centres de recherche, le niveau général des activités de R-D paraît très soutenu. Ce résultat s'explique d'abord par les caractéristiques du domaine pris en compte. Les firmes appartiennent à un ensemble technologique émergent, dont beaucoup de produits sont encore en développement, et marqué par le poids des marchés d'études de Défense. L'exemple de la firme P2 est particulièrement éloquent: avec un chiffre d'affaires de 100 millions de livres, la firme finance 4 millions de livres de travaux de R-D sur ses ressources propres et exécute des contrats d'études de Défense pour 20 millions de livres financés par ses clients. L'extensibilité de la notion de R-D peut aussi expliquer son niveau d'intensité élevé. Effectivement plusieurs entreprises évaluent une activité globale de recherche, développement et études, certaines précisant qu'il s'agit surtout d'études, dont il conviendrait d'ailleurs de pouvoir mieux cerner le contenu. L'évaluation des activités de recherche est une question récurrente, la classification utilisée ici est suffisamment large pour garder sa pertinence quant à la hiérarchie des efforts de R-D fournis par les entreprises, qui a par ailleurs été mise en rapport avec d'autres éléments recueillis au cours des entretiens.

\section{Présentation des différentes sources de connaissances externes}

Sur la base des résultats exposés dans la partie gauche du tableau 2 (colonnes $\mathrm{f}$ à $\mathrm{m}$ ), nous proposons une vue d'ensemble des collaborations liées aux problèmes d'innovation des entreprises de l'échantillon. Une analyse plus spécifique des relations avec les universités sera présentée à la section suivante. Ce bref tour d'horizon distingue, d'une part, les coopérations (à l'intérieur du groupe avec le centre de R-D, et à l'extérieur avec $\mathrm{d}$ 'autres entreprises, des laboratoires universitaires, des laboratoires publics

49. OCDE, Manuel d'Oslo, op. cit., p. 64. 
et des sociétés conseils) et, d'autre part, les financements (contrats d'études Défense, programmes gouvernementaux, contrats européens). Si la première catégorie comporte des flux financiers et la seconde suscite des coopérations, la distinction a cependant été faite parce que dans le premier cas, il s'agit de sources directes de connaissances scientifiques et technologiques, et dans le second cas de sources indirectes pour ces mêmes connaissances. Enfin, nous rappelons que les relations prises en compte concernent seulement les unités de production sélectionnées et non pas l'ensemble des groupes auxquels elles appartiennent.

\section{Relations avec le(s) centre(s) de $R-D$ du groupe}

Il existe encore beaucoup de laboratoires centraux de R-D au sein des groupes industriels à la fin des années 1990. Sur les 16 groupes qui intègrent une des unités de production de notre échantillon, 12 possèdent un ou plusieurs laboratoires de recherche, tandis que 8 entreprises de l'échantillon, sur les 12 appartenant à un groupe doté de centre(s) de R-D, entretiennent des relations étroites avec un centre de recherche interne au groupe. Ces liens de forte intensité existent sous des formes variées: relations marchandes entre des unités qui disposent d'une autonomie financière $(\mathrm{P} 2, \mathrm{P} 6, \mathrm{P} 9)$, ou relations hiérarchisées entre la recherche du centre et le développement lié à la production, ce qui n'exclut pas des échanges d'informations dans les deux sens (P1, P7, P11). La mobilité des ingénieurs entre le centre de recherche et l'unité de production renforce l'interdépendance des deux acteurs (P7). Au-delà de sa fonction de soutien pour la résolution des problèmes techniques des unités de production, le laboratoire central de R-D est un centre de ressources pour la formation de l'encadrement technique de l'ensemble de la firme. Les ingénieurs commencent souvent leur carrière par le centre de recherche qui joue ainsi un rôle de pépinière ${ }^{50}$.

Deux cas apparaissent comme plus spécifiques: celui d'une jointventure où il y a R-D partagée entre les équipes des deux partenaires (P5) et celui d'une unité aux activités de développement très limitées, mais dont les ingénieurs se déplacent très régulièrement au centre de recherche du

50. Pour plus d'informations sur la question des fonctions des centres de recherche des entreprises et en particulier leur rôle de pépinière de cadres techniques au compte de l'ensemble du groupe, on peut se reporter à J. P. Beltramo, J. J. Paul et C. Perret, "The recruitment of researchers and the organization of scientific activity in industry", International Journal of Technology Management, vol. 22, $\mathrm{n}^{\circ} 78,2001, \mathrm{p} .811-834$. 
groupe pour s'initier aux nouveaux produits et en retour contribuer à rendre les composants exploitables en production (P8). Parmi les 4 groupes qui, en plus de la petite entreprise indépendante (P16), ne possèdent pas de centre de recherche, 3 ont des structures plus financières qu'industrielles (holding ou conglomérat: $\mathrm{P} 10, \mathrm{P} 13, \mathrm{P} 15$ ) et le quatrième est de petite taille (P12). Une petite entreprise rachetée par un grand groupe disposant d'un laboratoire central n'a aucune relation avec celui-ci (P17), mais la collaboration se fait entre les deux sites de cette entreprise. Trois entreprises semblent échapper à la bipolarisation, fortes relations versus absence de relations avec un centre de R-D: la première (P3) n'a pratiquement pas d'activités de développement donc a peu de contacts avec le centre de R-D, la deuxième (P4) traite peu avec le centre de R-D, car ses activités de développement sont réduites et le groupe est organisé en unités financièrement autonomes, et la troisième (P14) appartient à un petit groupe doté seulement d'une petite unité de recherche stratégique. Enfin, il existe des coopérations intragroupes en dehors de celles qui lient les unités de production aux centres de recherche. Ces relations technologiques d'entreprise à entreprise au sein d'un groupe se vérifient en particulier lorsque la production est dirigée vers le marché interne (P1).

\section{Relations avec les autres entreprises (hors du groupe)}

Les collaborations se développent principalement avec les clients et les fournisseurs mais peuvent également intervenir entre entreprises non concurrentes (par exemple pour l'élaboration commune d'un sous-système où chacun des partenaires apporte ses compétences spécifiques). Par ailleurs, un fournisseur ou un client pour des composants peut être un concurrent au niveau des sous-systèmes. Ou encore, un partenaire technologique peut être racheté par un concurrent direct (dans ce cas - P2 l'entreprise considère que son intérêt est de maintenir l'accord technologique, parce que dans les autres domaines, où il y a concurrence avec le nouvel acquéreur, elle possède une avance qui la met à l'abri d'une menace directe à court terme). La concurrence n'exclut pas des partenariats limités à des domaines spécifiques entre entreprises concurrentes et, réciproquement, ces partenariats n'abolissent pas la concurrence dans les autres domaines. La concurrence peut se développer par le biais des relations entre clients et fournisseurs. Une entreprise française (P11) indique que lorsqu'elle commande des composants sensibles à des fournisseurs américains, ceux-ci mettent des conditions de divulgation de 
l'identité des clients de l'entreprise, ce qui les amène à connaître les marchés et parfois à essayer de récupérer les clients de l'entreprise. Les règles implicites de confidentialité ne suffisent pas toujours et parfois elles sont formalisées dans des accords (P15). Dans la mesure où il y a souvent plusieurs intermédiaires entre le composant fabriqué par une entreprise et le produit final qui l'incorpore, les entreprises sont en général très attentives aux marchés finaux de leurs clients, le marketing exerçant parfois un filtrage des relations avec les clients (P3).

Lorsqu'on examine les raisons qui font que 4 unités de production sur 17 n'ont pas de relations technologiques suivies avec d'autres entreprises hors de leur groupe, on peut les classer selon deux rubriques de nature très différente mais qui peuvent se combiner:

- la politique de secret industriel de l'entreprise (du groupe);

-- une production orientée vers le marché interne du groupe.

En revanche, il n'y a pas de relation évidente entre la standardisation des produits (qui de toute façon est limitée) et l'absence de liens technologiques. La confidentialité qui entoure les activités de développement est en relation avec la courte durée de vie de certains produits ( 2 à 3 ans) qui fait qu'il est essentiel d'arriver le premier sur le marché.

Le contenu technologique des relations interentreprises, c'est-à-dire pour l'essentiel des relations entre les clients et les fournisseurs, varie beaucoup. Ce n'est pas la fréquence des contacts qui engendre véritablement un échange de connaissances technologiques. Le critère essentiel paraît être l'accès à la R-D du client, du fournisseur ou du partenaire. Nous avons donc distingué les relations qui reposent seulement sur la spécification des produits, sous forme de contacts réguliers et de réunions de travail, et les coopérations qui impliquent un accès au laboratoire du partenaire (ces dernières pouvant aussi inclure des échanges sur la spécification des produits). Les entreprises qui collaborent pour la spécification des produits sont au nombre de quatre: P3, P9, P11 sont en relations suivies avec leurs clients et leurs fournisseurs, et P8, dont la production est vendue sur le marché interne, a cependant des partenariats avec des fournisseurs hors du groupe. Outre l'antagonisme entre la confidentialité de l'innovation et le partage du développement, un autre élément est avancé pour expliquer l'exclusion des activités de R-D de ce type de partenariat: les clients préfèrent ne pas participer à la résolution des 
problèmes liés à la mise au point des produits pour ne pas augmenter les coûts (P9).

On peut classer les coopérations technologiques selon les trois catégories suivantes:

\begin{tabular}{|l|c|}
\hline R-D partagée avec les clients ou les fournisseurs & $\mathrm{P} 5, \mathrm{P} 10, \mathrm{P} 12, \mathrm{P} 15$ \\
\hline $\begin{array}{l}\text { Partenariat technologique hors relations clients/ } \\
\text { fournisseurs }\end{array}$ & $\mathrm{P} 2, \mathrm{P} 6, \mathrm{P} 12, \mathrm{P} 14$ \\
\hline $\begin{array}{l}\text { Collaboration mise en œuvre par un programme } \\
\text { institutionnel }\end{array}$ & $\mathrm{P} 13, \mathrm{P} 16$ \\
\hline
\end{tabular}

La recherche conjointe, par le client et son fournisseur, de solutions aux problèmes posés par la mise au point d'un produit et le travail en commun des ingénieurs au sein des laboratoires des deux protagonistes s'étendent parfois de la conception jusqu'aux tests, en passant par le développement. Le produit est ainsi réalisé en partenariat avec le client. Lorsque l'entreprise n'appartient pas à un groupe doté d'un centre de recherche, les grands clients peuvent représenter une source de R-D particulièrement importante (P10, P12). Pour ce faire, devenir partenaire de grands acteurs mondiaux des télécommunications devient une stratégie explicite (P12). Les partenariats avec les entreprises autres que les clients et les fournisseurs sont soit à l'initiative de l'établissement lui-même, soit s'inscrivent dans un accord passé au niveau de la société mère. Dans un cas, le partenariat se traduit par la mise en place d'une structure mixte permanente, qui inclut la participation d'un laboratoire public. Les deux programmes qui ont généré des collaborations avec d'autres entreprises sont l'un goulvernemental (britannique) et l'autre européen. Pour le contrat européen, il s'agit de recherche plus en amont avec des partenaires industriels et universitaires. Enfin, il faut aussi mentionner les opérations de sous-traitance qui ne se confondent pas avec le codéveloppement. La sous-traitance de R-D peut intervenir dans le cadre d'un partenariat technologique sans pour autant être systématiquement utilisée dans ce type de relations et inversement, sous-traiter de la R-D n'implique pas nécessairement une coopération des ingénieurs des deux contractants. 
146 L'économie du savoir: une économie de la collaboration?

\section{Relations avec les universités}

Le secteur des universités comprend le CNRS, selon la convention adoptée par l'OCDE. La très grande majorité des unités de production ont noué des liens directs de recherche et de développement avec des universités. Il existe également des relations, qui ne seront pas traitées ici, plus spécifiquement centrées sur l'enseignement et le recrutement. Cela est surtout vrai pour les entreprises britanniques qui recrutent leurs ingénieurs dans les universités, contrairement à la situation française où les écoles d'ingénieurs représentent la filière principale d'embauche. Pour cette raison et parce que les pratiques d'intervention des entreprises dans l'enseignement supérieur diffèrent en France et en Grande-Bretagne, où par exemple des chaires sont financées par des firmes, les objectifs liés à l'enseignement pèsent plus au Royaume-Uni dans les relations avec les universités.

On a noté plus haut la persistance, à la fin des années 1990, de l'organisation des activités de R-D dans des unités distinctes de la production, au sein de beaucoup de groupes industriels. Le centre de recherche par son activité a des liens avec les universités, mais il est souvent aussi le centre organisateur (en liaison avec le siège) de l'ensemble des relations du groupe avec la recherche universitaire. Cette centralisation est plus ou moins forte, de l'institution d'un responsable des collaborations extérieures de R-D et du simple contrôle des contrats, à une administration mondiale qui comprend l'identification des universités et organismes publics ressource, ainsi que la maitrise des accords cadres avec les partenaires universitaires et la gestion de la propriété intellectuelle qui en découle. Mais ce n'est pas dans tous les cas où il y a des liens forts entre l'unité de production et le centre de recherche que ce dernier est le passage obligé pour les relations avec les universités. Le centre de recherche peut aussi simplement orienter vers un laboratoire universitaire l'établissement qui le sollicite.

Une seule entreprise (P8) n'a pas de contacts avec les universités: elle produit pour son groupe et l'appui lui est fourni par le centre de recherche qui, par ailleurs, est en relation avec des laboratoires universitaires. Pour six entreprises, les contacts avec les universités se limitent à des relations de services (P1, P14), en particulier d'utilisation d'équipement (P10), et de consultance $(\mathrm{P} 3, \mathrm{P} 15)$ ou encore à des collaborations non contractuelles (P7). L'évolution d'une de ces entreprises (P14) mérite d'être rapportée. Sur une décennie, elle a connu un double désengagement de ses 
collaborations de R-D, d'une part, avec ses clients militaires et, d'autre part, avec les universités. Le premier désengagement est lié à la baisse des commandes de la Défense qui a fait chuter la part des produits militaires de 65 à $15 \%$ en dix ans, et le second est à la fois une conséquence du premier dans la mesure où la réalisation des contrats du ministère de la Défense impliquait des collaborations avec les universités, et à la fois le résultat d'une politique délibérée. L'ancienne approche qui associait une recherche interne de type «blue $s k y^{51}$ » et des liens très étroits avec une université (financement d'une chaire d'optoélectronique et de doctorants) a été totalement abandonnée pour faire place à une activité de conception et de développement très proche du marché. L'effondrement des contrats de la Défense à «coûts majorés» qui permettaient de financer les recherches en amont et la faiblesse des résultats commerciaux de la recherche «blue sky» expliquent ce changement radical de stratégie.

Si une minorité d'entreprises de l'échantillon a seulement des relations de services avec les universités, en revanche la majorité a des relations de recherche (qui se combinent assez souvent avec des relations de consultance ou d'utilisation des équipements universitaires). Le financement d'un doctorant est la forme dominante des relations contractuelles de recherche. Sur dix entreprises ayant des relations formalisées de recherche avec des universités, huit financent des doctorants ou post-doctorants. Pour six de ces entreprises, c'est la seule forme de contrat qui les lie aux universités et même pour deux d'entre elles, la seule forme de relation avec les universités. Dans la plupart des cas, le doctorant fait sa thèse à l'université sur un sujet qui intéresse l'entreprise. C'est une façon pour cette dernière de soutenir de la recherche exploratoire, avec une incertitude quant aux possibilités d'utiliser les résultats pour ses produits mais pour un coût faible, puisque l'entreprise ne paie qu'une partie d'une rémunération bien en deçà du salaire d'un ingénieur de $R-D^{52}$.

51. Terme souvent utilisé dans l'industrie pour désigner des travaux de recherches génériques, non directement liés à la mise sur le marché d'un produit spécifique.

52. Les dispositifs de thèse en entreprise sont organisés dans le cadre des Conventions industrielles de formation par la recherche (CIFRE) en France et des Cooperative Awards in Science and Engineering (CASE) en Grande-Bretagne. Les conventions CIFRE associent trois partenaires: une entreprise qui embauche sur contrat à durée déterminée ou indéterminée, un diplômé d'école d'ingénieur, de commerce ou d'université possédant un diplôme d'études approfondies (DEA) et un laboratoire d'accueil (d'université, d'école d'ingénieurs ou d'organisme de recherche publique) qui assure l'encadrement de la thèse. La rémunération du jeune cadre - doctorant est partagée entre l'État qui participe de manière forfaitaire pour un montant égal à la moitié du coût salarial plancher d'un ingénieur pendant trois ans et l'entreprise qui 
L'orientation du travail du doctorant est plus ou moins marquée: l'entreprise se contente parfois d'indiquer le domaine de recherche qui l'intéresse sans fixer d'objectifs de résultats spécifiques (P5), alors que dans d'autres cas, la thèse correspond à un projet de l'entreprise qui en contrôle l'avancement (P12, P13, P17). Mais le financement d'un post-doc peut apparaître mieux adapté à la réalisation d'un projet spécifique de l'entreprise que le financement d'un doctorant (P9). Le doctorant est aussi utilisé en dehors de son sujet de thèse pour des travaux de recherche amont rémunérés $(\mathrm{P} 13, \mathrm{P} 17)$.

\section{Relations avec les laboratoires publics ou indépendants}

On peut voir un effet de la différence des systèmes nationaux d'innovation britannique et français dans la totale dissymétrie qui caractérise les liens, d'une part, entre les établissements industriels et les laboratoires à financement public implantés sur le territoire britannique et, d'autre part, les établissements et les laboratoires publics sur le territoire français. Dans le cas britannique, une seule entreprise sur dix (P4) a des relations avec un laboratoire à financement public, et il faut préciser qu'il s'agit de relations de services (tests, caractérisation, expertise), alors que dans le cas français, l'ensernble des entreprises a des relations de collaboration avec des laboratoires publics. Le recours à des organismes indépendants n'est pas un substitut, puisqu'une seule entreprise britannique (P13) utilise leurs services de R-D, et aucune entreprise française.

Cette situation très contrastée entre les deux pays s'explique par les changements institutionnels respectifs au sein des organismes publics de recherche, au cours des années 1990. La privatisation ou la réduction du financement public des organismes de recherche publics spécialisés a été plus précoce et plus marquée en Grande-Bretagne qu'en France. Le changement de statut ou la réduction des financements institutionnels des

assure au moins l'équivalent. Les CASE awards (et leur variante, les Industrial CASE) suivent le même principe de cotutelle: un boursier préparant un $\mathrm{PhD}$ travaille sur un projet supervisé par un responsable académique et un responsable industriel. Il est prévu que l'étudiant travaille dans le laboratoire industriel au minimum trois mois et au maximum dix-huit mois, sur trois années. La participation financière de l'entreprise est plus réduite que dans le cas français et peut varier avec les disciplines: à titre d'exemple, un étudiant financé par l'EPSRC (Engineering and Physical Science Research Council) reçoit (au minimum) une bourse annuelle de 9000 livres et une contribution de 3000 livres du partenaire industriel, qui verse également 1400 livres par an au département universitaire. 
centres de recherche britanniques les a contraints à vendre leurs services à prix coûtant et à limiter leurs contacts avec les universités, rompant ainsi une chaîne où les universités alimentaient en recherche amont des centres spécialisés dans des applications spécifiques susceptibles d'être utilisées par les entreprises du secteur industriel correspondant, moyennant de faibles redevances couvrant les coûts de fonctionnement. Les organismes scientifiques et technologiques indépendants ne sont pas plus utilisés, car soumis aux mêmes contraintes du marché, ils ont des prestations très onéreuses et n'ont pas les moyens de travailler avec les universités pour rester en phase avec les avancées scientifiques. En France, pour les domaines qui concernent les entreprises de notre étude et pour la période d'observation (1997, ainsi que les années qui précèdent), deux organismes publics jouent un rôle déterminant: France Télécom et son centre de recherche, le CNET, ainsi que le Laboratoire d'électronique, de technologie et d'instrumentation (LETI) du Commissariat à l'énergie atomique (CEA).

Le schéma de base de l'intervention de France Télécom auprès des entreprises est le suivant: France Télécom finance un marché d'études au bénéfice d'une entreprise qui développe un projet dans lequel un laboratoire $d \mathfrak{u}$ CNET intervient comme expert, en apportant ses connaissances. Jusqu'à la fin des années 1990, le CNET a tenu un rôle d'intermédiaire entre la recherche universitaire à long terme, avec laquelle il entretenait des liens importants, et la recherche industrielle à court terme. En revanche, le rôle de centre technique prévu à l'origine sous forme de réponses aux demandes des entreprises n'a pas été réellement tenu. L'intervention du CNET s'est réduite au cours des années 1990: le processus de privatisation de France Télécom a accéléré le phénomène ${ }^{53}$, mais la cause initiale tient au fait que les partenariats industriels mis en œuvre par France Télécom ont permis la constitution d'équipe de R-D au sein des entreprises, alors qu'elles n'existaient pas pour un domaine nouveau tel que l'optoélectronique. Ces nouvelles équipes de recherche industrielle se sont créées par apprentissage auprès des équipes du CNET, mais aussi plus directement par transfert d'ingénieurs du CNET. La transformation du service public des télécommunications en société anonyme a réorienté la R-D de France Télécom vers les besoins propres de

53. Jusqu'au 31 décembre 1990, le service public des télécommunications était un monopole d'État, puis le $1^{\text {er }}$ janvier 1991 a été créé l'exploitant public «France Télécom» et le $1^{\text {er }}$ janvier 1997 l'entreprise nationale France Télécom a été transformée en société anonyme détenue majoritairement par l'État. 
l'entreprise, le CNET devenant France Télécom R-D le $1^{\text {er }}$ mars 2000, et n'assumant plus la fonction de soutien à la recherche industrielle des équipementiers du secteur des télécommunications. Cinq des sept entreprises de l'échantillon localisées en France ont bénéficié du partenariat institutionnel de France Télécom et du CNET: les quatre qui opèrent sur les marchés des télécommunications (P7, P8, P9 et P10) ainsi qu'une cinquième (P6) dont l'activité se divise entre les produits militaires et les produits civils (dont une minorité d'application pour les télécommunications) mais dont la maison mère était un grand partenaire de France Télécom et du CNET.

Les deux autres entreprises françaises (P11 et P17) collaborent avec le LETI, la seconde étant une ancienne start-up de cet organisme. Laboratoire d'électronique créé en 1968 au sein du CEA, organisme public de recherche spécialisée, le LETI doit trouver une partie de ces moyens, y compris les salaires, par contrats externes, dont une partie auprès d'organismes publics ou de l'Union européenne. Tous les développements sont orientés vers le transfert industriel. Trois modalités de transfert peuvent se présenter:

i. transfert à l'industriel, sous forme d'accord incluant un contrat d'exclusivité pour l'exploitation d'un brevet (comme c'est le cas avec P11),

ii. création d'une société en joint-venture avec un partenaire industriel,

iii. création d'une filiale sur le capital du CEA ou d'une start-up avec un ingénieur qui quitte le LETI. Lorsque l'entreprise créée tourne, le CEA se dégage. Pour P17, le projet initial a été un échec, le CEA s'est retiré du capital, mais les liens étroits avec le LETI se sont maintenus. Dans le cas de l'accord particulier entre le LETI et P11, la collaboration est permanente pour entreprendre des recherches exploitables sur le marché. Mais il faut noter que seules les grandes entreprises peuvent acheter la recherche du LETI, les PME ou les établissements appartenant à des groupes de moyenne importance doivent obtenir des aides gouvernementales pour pouvoir en assumer le coût (P11 a bénéficié d'un financement de l'Agence nationale de valorisation de la recherche - ANVAR). L'appartenance du LETI au CEA lui permet de bénéficier de son infrastructure et de contacts avec les laboratoires de recherche fondamentale de l'organisme public ainsi qu'avec des 
équipes mixtes CEA-CNRS. Hors du CEA, le LETI passe également des accords pour la recherche amont avec le CNRS et les universités.

\section{Sources de financement de la $R-D$ et aides gouvernementales}

Les relations avec les universités et les laboratoires publics induisent un financement public de la R-D et certaines collaborations interentreprises bénéficient également d'un support public, tandis que les clients représentent une source essentielle de financement de la R-D.

La première motivation de l'entreprise pour entreprendre de la R-D est que le client la paye, son but n'étant pas de faire de la recherche, mais du profit. L'entreprise peut d'ailleurs passer d'une activité basée sur le laboratoire à la grande production manufacturière (P14). Cette évolution a cependant des limites, dans la mesure où la production de masse en Europe est concurrencée par celle des sites délocalisés où le coût de la maind'œuvre est plus faible. Une autre stratégie consiste à lancer le développement d'un nouveau produit seulement après que son financement a été obtenu auprès du client (P14), l'étude des propriétés de base, la caractérisation et le développement du produit pouvant par ailleurs être sous-traités à un laboratoire universitaire (P13). L'ensemble des organismes publics et privés finançant les travaux de R-D ou d'innovation sont autant de sources externes indirectes de connaissances scientifiques et technologiques.

Malgré une réduction sensible au cours des années 1990, les contrats d'études du ministère de la Défense, en France comme en GrandeBretagne, représentent la première source de financement public de la R-D des entreprises de 1'échantillon ${ }^{54}$. Près de la moitié des entreprises ( 8 sur 17 ) en bénéficient. C'est le cas des cinq entreprises qui vendent au moins

54. La chute des marchés militaires au début des années 1990 a eu un fort impact sur l'activité de la plupart des firmes, posant la question de leur reconversion. Celle-ci est plus orientée vers la recherche de nouvelles applications civiles pour des lignes de produit existantes que vers une véritable reconversion dans la conception de produits dans des domaines nouveaux. Les tentatives d'entreprises américaines dans cette direction ont globalement échoué. Conjointement à la diminution de la demande de produits militaires, il y a eu réduction des marchés d'études militaires. La baisse de la principale source extérieure de financement de la R-D a eu des conséquences sur l'ensemble des produits, au-delà de la seule conception des composants et systèmes optoélectroniques. Les contrats d'études militaires sont un moyen essentiel de financement de la recherche à moyen terme pour la majorité des unités de production, y compris celles dont la production n'est pas principalement destinée aux marchés militaires. 
la moitié de leur production sur les marchés militaires (P2, P5, P6, P9 et P11), mais également de trois entreprises dont les produits à usage militaire ne représentent qu'entre 15 et $20 \%$ des ventes (P12, P14 et P17).

L'aide gouvernementale à l'innovation, hors défense, ne concerne que quelques entreprises et selon des modalités propres à chaque pays. L'ANVAR est l'organisme financeur pour les deux entreprises françaises bénéficiaires (P11 et P12). L'Agence française de l'innovation soutient en particulier des transferts de technologie, des projets innovants et des recrutements pour l'innovation. Selon le scénario indiqué plus haut, elle peut intervenir en soutien financier d'un projet de recherche entrepris par un laboratoire public pour le compte d'une entreprise, lorsque celle-ci se déclare dans l'incapacité d'en assumer le coût (P11). Plusieurs procédures gouvernementales de soutien à l'innovation ont été utilisées par les entreprises en Grande-Bretagne: LINK scheme, Teaching Company Scheme (TCS) et Small firms Merit Award for Research and Technology $(\text { SMART })^{55}$. Mais au moment de l'enquête une seule firme bénéficiait d'un financement SMART (P16). Une firme avait présenté un projet LINK, mais avait échoué (P9). Une autre (P14) n'avait pas renouvelé son association à un projet LINK, jugeant ses résultats trop éloignés du marché. Deux entreprises ayant eu recours à des recrutements financés par les TCS se sont désengagées de ce type de programmes, l'une préférant par souci d'efficacité embaucher elle-même sur un projet de l'entreprise (P9), tandis que l'autre dit avoir eu une expérience très négative, parce que l'équipe universitaire qui encadrait le diplômé embauché faisait ce qu'elle voulait, et pas ce que voulait l'entreprise (P2).

Le recours à des programmes européens est également limité. Trois unités de production (P12, P13 et P17) bénéficient d'un financement européen en 1997. Une quatrième (P10) a participé antérieurement à un programme européen mais n'a pas donné de suite, car il n'y a pas eu de débouchés industriels - «l'entreprise n'est pas une entreprise de

55. Les programmes LINK sont les principaux mécanismes gouvernementaux au Royaume Uni pour la promotion de la recherche précommerciale en partenariat, associant des entreprises et des laboratoires de recherche. Ils fournissent le financement de $50 \%$ de la recherche précompétitive et des investissements nécessaires au succès de la commercialisation des nouvelles technologies. La spécificité des TCS - remplacés par les Knowledge Transfer Partnerships depuis juin 2003 - qui associent également recherche de base et entreprises, est d'affecter la majorité du financement au recrutement de diplômés pour travailler sur les projets. Les soutiens SMART sont destinés aux individus et aux petites entreprises pour exécuter un microprojet, une étude de faisabilité ou un projet de développement. 
recherche». Pour deux autres unités de production (P1 et P5), c'est le centre de R-D qui participe à un programme européen. Le jugement sur les programmes européens est parfois très négatif: «c'est un échec, car il faut un schéma industriel préalable; il ne s'agit pas de coopérer pour rien; la recherche partagée des programmes européens ne produit rien, ce n'est qu'au niveau du développement, où le partage n'est pas possible pour des raisons de secret qu'il y aurait efficacité à coopérer» (P7). Une autre entreprise (P9) avance des arguments proches de ceux de (P10): les financements européens demandent trop de démarches et de compromis avec les partenaires, alors que les programmes semblent plus concerner des équipes de recherche. Une des entreprises participantes (P12) confirme a contrario cette appréciation négative, en justifiant sa participation aux programmes européens par sa position de leader qui lui a permis d'orienter les recherches vers ses propres objectifs de marché.

\section{Les caractéristiques de la demande de connaissances des unités de production et la place de la recherche universitaire}

La variété de l'effort d'innovation au sein de l'échantillon ayant été mise en évidence ainsi que la diversité des sources de connaissances et de la nature des coopérations, nous allons dans, un premier temps, rechercher les liens éventuels entre structures d'innovation et sources de connaissances.

\section{Mode d'innovation et relations de recherche des unités de production}

\section{Types élémentaires d'innovation}

Nous opérons une classification des unités de production selon des types d'innovation définis sommairement à partir de l'intensité de la R-D et des formes d'organisation de la recherche au sein des structures auxquelles appartiennent les établissements. En l'absence d'une mesure directe de l'innovation, suffisamment fréquente et fiable ${ }^{56}$, on utilise donc l'intensité de la R-D comme indicateur de l'intensité de l'innovation. Nous avons distingué trois formes d'organisation: la structure de groupe centralisée avec centre de recherche, la structure de groupe décentralisée

56. Voir les remarques de la section 3.2 sur la difficulté à obtenir un indicateur d'innovation à l'aide de la notion de produits nouveaux. On peut ajouter que le critère des brevets déposés serait encore moins opérationnel pour notre échantillon. 
sans centre de recherche et l'entreprise indépendante. Il n'y a pas de stricte équivalence entre l'existence d'un centre de recherche et une organisation centralisée de la R-D, mais nous avons déjà noté que dans les groupes organisés en divisions autonomes, y compris les centres de R-D, il existe des échanges privilégiés et importants entre centres de recherche et unités de production, la différence avec les structures où il n'y a pas d'autonomie des divisions portant essentiellement sur les modalités de facturation de ces échanges. En revanche, par définition, on ne peut pas concevoir des relations de recherche verticale au sein des groupes où il n'existe pas de centre de R-D. Le croisement du niveau de R-D et de la structure organisationnelle de la recherche donne la répartition suivante des entreprises:

\begin{tabular}{|l|c|c|c|}
\hline & $\begin{array}{c}\text { Structure de groupe } \\
\text { centralisée } \\
\text { (avec centre de } R-D)\end{array}$ & $\begin{array}{c}\text { Structure de groupe } \\
\text { décentralisée } \\
\text { (sans centre de } R-D)\end{array}$ & $\begin{array}{c}\text { Entreprise } \\
\text { indépendante }\end{array}$ \\
\hline$R-D$ faible & $\begin{array}{c}\mathrm{P} 3, \mathrm{P} 4, \mathrm{P} 8, \\
\mathrm{P} 13, \mathrm{P} 14, \mathrm{P} 17\end{array}$ & - & - \\
\hline$R-D$ moyenne & $\mathrm{P} 1, \mathrm{P} 5, \mathrm{P} 6$, & $\mathrm{P} 10, \mathrm{P} 12$, & $\mathrm{P} 16$ \\
$\mathrm{P} 9, \mathrm{P} 11$ & - & - \\
\hline$R-D$ forte & $\mathrm{P} 2$, & - & \\
\hline
\end{tabular}

En groupant R-D moyenne et R-D forte et structure de groupe décentralisée et entreprise indépendante, on en déduit trois types élémentaires d'activité d'innovation:

- Type I: unités de production à faible intensité de R-D, appartenant à un groupe doté d'un centre de R-D (six entreprises: P3, P4, P8, P13, P14 et P17).

- Type II: unités de production à intensité de R-D moyenne ou forte, appartenant à un groupe doté d'un centre de R-D (sept entreprises: P1, P2, P5, P6, P7, P9 et P11).

- Type III: unités de production à intensité de R-D moyenne, appartenant à une structure de groupe sans centre de R-D ou constituant une structure indépendante (quatre entreprises: P10, P12, P15 et P16). 
Puisque toutes les unités de production à faible intensité de R-D appartiennent à un groupe doté d'un centre de R-D, on peut se demander s'il n'y a pas une division du travail entre les unités de production et le centre de recherche, et si ce dernier ne concentre pas toutes les activités de R-D. La réponse est entièrement négative. D'une part, plus de la moitié des entreprises appartenant à un groupe doté d'un centre de R-D font au moins autant de recherche que celles qui ne sont pas dans une structure avec centre de R-D. D'autre part, comme l'indique le tableau suivant, les entreprises qui font peu de recherche ont également peu de liens avec le centre de R-D de leur groupe:

\begin{tabular}{|c|c|c|c|}
\hline & $\begin{array}{c}\text { Pas de liens avec le } \\
\text { centre de R-D }\end{array}$ & $\begin{array}{c}\text { Liens faibles } \\
\text { avec le centre } \\
\text { de } R-D\end{array}$ & $\begin{array}{c}\text { Liens forts } \\
\text { avec le centre } \\
\text { de } R-D\end{array}$ \\
\hline $\begin{array}{c}\text { Établissements } \\
\text { de type I } \\
(R-D \text { faible })\end{array}$ & 2 & 3 & 1 \\
\hline $\begin{array}{c}\text { Etablissements } \\
\text { de type II } \\
(R-D \text { moyenne et } \\
\text { forte })\end{array}$ & 0 & 0 & 7 \\
\hline
\end{tabular}

Ces chiffres confirment la complémentarité des centres de recherche et des unités de production pour ce qui concerne la R-D. Toutes les entreprises qui ont un niveau moyen ou élevé de R-D ont des relations étroites avec leur centre de recherche. Inversement, une seule entreprise parmi celles qui font peu de développement coopère fortement avec son centre de R-D. La recherche qui n'est pas faite au niveau de l'unité de production n'est pas exécutée par le laboratoire central. Il y a bien une spécificité des travaux de R-D mis en œuvre par les unités de production.

$\mathrm{Ce}$ premier résultat intéressant suscite d'autres questions. Les entreprises à faible intensité de R-D qui n'utilisent pas, ou qui utilisent peu, les services cles centres de R-D de leur groupe ont-elles recours à d'autres sources de connaissances technologiques? Et a contrario pour les entreprises dont l'intensité de R-D est plus forte, le centre de recherche remplace-t-il les sources externes de connaissances? Par ailleurs, l'absence d'un centre de R-D au sein du groupe ou le fait d'être une entreprise 
156 L'économie du savoir: une économie de la collaboration?

indépendante (type III) influencent-ils l'acquisition de connaissances externes?

\section{Types d'innovation et relations de recherche}

Les liens respectifs avec les autres entreprises, les universités et les laboratoires publics pour les entreprises à faible intensité de recherche (type I), à intensité de recherche renforcée par un centre de R-D (type II) et à intensité de recherche moyenne (type III) sont présentés ci-dessous :

\begin{tabular}{|c|c|c|c|}
\hline & $\begin{array}{l}\text { Liens autres } \\
\text { entreprises }\end{array}$ & Liens universités & Liens laboratoires publics \\
\hline $\begin{array}{l}\text { Type I } \\
\text { (R-D faible) }\end{array}$ & $\begin{array}{l}\text { Forts : } 2 \\
\text { Faibles / nuls : } 4\end{array}$ & $\begin{array}{l}\text { Forts : } 3 \\
\text { Faibles / nuls : } 3\end{array}$ & $\begin{array}{l}\text { Forts : } 2 \\
\text { Faibles / nuls : } 4\end{array}$ \\
\hline $\begin{array}{l}\text { Type II } \\
\text { ( } R-D \text { liée à un } \\
\text { centre) }\end{array}$ & $\begin{array}{l}\text { Forts : } 3 \\
\text { Faibles / nuls : } 4\end{array}$ & $\begin{array}{l}\text { Forts : } 5 \\
\text { Faibles / nuls : } 2\end{array}$ & $\begin{array}{l}\text { Forts : } 3 \\
\text { Faibles / nuls : } 4\end{array}$ \\
\hline $\begin{array}{l}\text { Type III } \\
\text { (R-D } \\
\text { moyenne) }\end{array}$ & $\begin{array}{l}\text { Forts : } 4 \\
\text { Faibles / nuls : } 0\end{array}$ & $\begin{array}{l}\text { Forts : } 2 \\
\text { Faibles / nuls : } 2\end{array}$ & $\begin{array}{l}\text { Forts : } 2 \\
\text { Faibles / nuls : } 2\end{array}$ \\
\hline
\end{tabular}

Toutes les entreprises qui n'appartiennent pas à un groupe doté d'un centre de recherche (type III) ont des relations fortes avec les autres entreprises, c'est-à-dire pour l'essentiel leurs clients. En revanche, pour les entreprises de type I et II, les relations technologiques interentreprises faibles l'emportent sur les relations fortes. Cela suggère qu'il pourrait $y$ avoir substitution entre la R-D des clients et le laboratoire central du groupe. Cette idée se trouve confirmée par le fait, déjà signalé, que l'accès à la R-D des grands clients est une stratégie pour des entreprises qui ne sont pas reliées à un centre de recherche interne à leur groupe. En revanche, il y aurait plutôt complémentarité des centres de recherche des entreprises et des laboratoires universitaires. En effet c'est seulement au sein du groupe des entreprises de type II, c'est-à-dire reliées à un centre de recherche interne à leur groupe, que les relations fortes avec les universités sont dominantes. La double indication d'une substitution clients/centres de R-D et d'une complémentarité universités/ centres de R-D est conforme aux hypothèses initiales et conforte la thèse de la spécificité de la R-D industrielle par rapport à la recherche universitaire. 
Dans la mesure où les liens forts avec les laboratoires publics concernent les seuls établissements situés sur le territoire français, la colonne relative aux relations avec les laboratoires publics représente les distributions respectives des établissements de France et du Royaume-Uni selon la structure d'innovation. Les laboratoires publics sont perçus, aussi bien par leurs responsables que par les entreprises, comme occupant une position intermédiaire entre la recherche industrielle et la recherche universitaire. La complémentarité, plutôt que la substitution, entre les laboratoires publics et la recherche universitaire, se trouve confirmée, par les informations complémentaires qui suivent. Parmi les sept entreprises (françaises) ayant des liens forts avec les laboratoires publics, quatre ont également des liens forts avec les universités, ce qui est comparable avec la structure des relations entre les universités et les entreprises britanniques (qui ont peu ou pas de liens avec les laboratoires publics), soit six liens forts contre quatre liens faibles ou nuls. En revanche, en l'absence de laboratoire central au sein du groupe, le laboratoire public peut constituer un substitut de celui-ci: c'est le rôle qu'attribue l'entreprise P10 au CNET.

Le mode d'innovation des entreprises doit aussi être mis en rapport avec une autre forme fondamentale de partenariat, les contrats d'études de défense qui ont été définis comme la principale source externe indirecte de connaissances scientifiques et technologiques. Les cinq entreprises dont les produits sont majoritairement destinés aux marchés militaires ont une intensité de R-D moyenne ou élevée. Comme le montre le tableau suivant, les contrats d'études militaires sont concentrés dans les entreprises des grands groupes qui bénéficient de relations de recherche fortes, à l'intérieur du groupe et avec les universités (type II):

\begin{tabular}{|c|c|c|c|}
\hline & $\begin{array}{l}\text { Liens autres } \\
\text { entreprises }\end{array}$ & Liens universités & $\begin{array}{l}\text { Contrats d'études } \\
\text { Défense }\end{array}$ \\
\hline $\begin{array}{l}\text { Type I } \\
\text { (R-D faible) }\end{array}$ & $\begin{array}{l}\text { Forts : } 2 \\
\text { Faibles / nuls : } 4\end{array}$ & $\begin{array}{l}\text { Forts : } 3 \\
\text { Faibles / nuls : } 3\end{array}$ & $\begin{array}{l}\text { Oui : } 2 \\
\text { Non : } 4\end{array}$ \\
\hline $\begin{array}{l}\text { Type II } \\
\text { (R-D liée à } \\
\text { un centre })\end{array}$ & $\begin{array}{l}\text { Forts : } 3 \\
\text { Faibles / nuls : } 4\end{array}$ & $\begin{array}{l}\text { Forts : } 5 \\
\text { Faibles / nuls : } 2\end{array}$ & $\begin{array}{l}\text { Oui : } 5 \\
\text { Non : } 2\end{array}$ \\
\hline $\begin{array}{l}\text { Type III } \\
(R-D \\
\text { moyenne })\end{array}$ & $\begin{array}{l}\text { Forts : } 4 \\
\text { Faibles / nuls : } 0\end{array}$ & $\begin{array}{l}\text { Forts : } 2 \\
\text { Faibles / nuls : } 2\end{array}$ & $\begin{array}{l}\text { Oui : } 1 \\
\text { Non : } 3\end{array}$ \\
\hline
\end{tabular}

Cette présentation précise la classification que nous avons esquissée. Les entreprises de type I, dont le niveau d'activité de R-D est inférieur à la 
moyenne, et qui n'utilisent pas les capacités du centre de recherche de leur groupe, peuvent être autosuffisantes pour leurs besoins de recherche, ou bien limiter leurs contrats de recherche externe par manque de moyens financiers. Les entreprises de type II, qui appartiennent aux plus grands groupes, et ont un niveau d'activité de R-D supérieur ou égal à la moyenne, renforcé par les relations avec le centre de recherche du groupe, bénéficient plus fréquemment que les autres entreprises, à la fois des résultats de la recherche universitaire et de possibilités de recherche financées par le ministère de la Défense. Les entreprises de type III qui n'appartiennent pas à des structures dotées d'un centre de recherche, et ont un niveau d'activité de R-D moyen, privilégient les relations de recherche avec les autres entreprises, principalement leurs clients.

La présomption d'un lien entre l'existence de contrats avec les universités et le bénéfice de financements de la Défense se trouve confirmée, lorsqu'on croise, pour l'ensemble des entreprises, la qualité des relations avec les universités avec la présence de contrats d'études:

\begin{tabular}{|c|c|c|c|}
\hline & \multicolumn{2}{|c|}{ Financements Défense } \\
\hline & & Contrats d'études & $\begin{array}{l}\text { Pas de contrats } \\
\text { d'études }\end{array}$ \\
\hline \multirow{2}{*}{$\begin{array}{l}\text { Liens } \\
\text { universités }\end{array}$} & Forts & 7 & $\begin{array}{r}3 \\
\end{array}$ \\
\hline & $\begin{array}{l}\text { Nuls ou } \\
\text { faibles }\end{array}$ & 1 & 6 \\
\hline
\end{tabular}

Des entreprises qui ne produisent pas pour les marchés militaires peuvent avoir des relations fortes avec les universités. En revanche, il est exceptionnel qu'un contrat d'études militaires ne soit pas accompagné de relations contractuelles avec les universités. En effet, nous avons vu que les entreprises sous-traitent les travaux de recherche militaires aux universités et que les contrats d'études sont des sources essentielles de financement de la recherche, aussi bien interne qu'externe. Le cas qui semble faire exception a déjà été évoqué: il s'agit de l'entreprise P14 qui s'est dégagée de ses relations contractuelles avec les universités et s'est réorientée vers un développement très proche du marché alors que ses commandes militaires étaient en chute libre. 


\section{Les attitudes des unités de production vis-à-vis de la recherche univer- sitaire}

À une exception près, toutes les entreprises ont des relations avec les universités, mais les liens restent limités puisque pour l'essentiel ils se traduisent pour les entreprises, par le recours - à titre gratuit ou oné reux - à des services des laboratoires universitaires ou à l'accueil de doctorants. Lors des entretiens, des difficultés d'accès aux universités ont été signalées mais également des réticences à recourir à la recherche universitaire. Il n'est pas inutile de mentionner ces obstacles à la collaboration, avant d'examiner dans quelle mesure les informations recueillies sur les coopérations entre les entreprises et les universités sont conformes aux hypothèses initiales.

\section{Les facteurs limitatifs de l'accès à la recherche universitaire}

(i) Les conséquences du rôle accru de l'innovation de procédés:

Le déplacement de la concurrence des produits vers les procédés ( $c f$. 3.2) peut réduire les demandes vis-à-vis des universités dans la mesure où l'accès à la recherche est plus problématique pour les procédés que pour les produits, comme le mentionne le responsable de l'entreprise P17:

L'entreprise n'a pas besoin de faire des propositions aux universitaires sur les besoins en produits nouveaux, car ils les connaissent et peuvent donner les réponses. En revanche, pour les procédés, l'entreprise aurait des demandes que ne connaissent pas les laboratoires, mais l'entreprise ne tient pas à dévoiler ses procédés.

On doit préciser que la réticence à consulter les laboratoires universitaires pour l'amélioration des procédés n'existe pas pour d'autres entreprises (P9, P11).

(ii) La question du risque et de la rentabilité de l'investissement de $R-D$ :

Le manque de ressources pour le financement de la recherche externe est fréquemment mis en avant comme obstacle au développement des coopérations avec la recherche universitaire. Les collaborations sont limitées à un seul partenariat, faute de moyens financiers (P6). La recherche de contacts avec les équipes universitaires se double d'une 
160 L'économie du savoir: une économie de la collaboration?

recherche de subventions, sous forme d'avances remboursables (P11). Les contrats de recherche ne sont passés avec les universités que lorsque le financement a été trouvé auprès d'un client (P13). La chute des contrats d'études militaires a tari les relations avec les universités (P14). En revanche, les demandes d'expertise, en particulier d'analyse de surface, sont souvent adressées à un laboratoire universitaire de préférence à une société spécialisée, parce qu'elles sont moins coûteuses (P13). L'insuffisance des moyens financiers disponibles pour accéder à la recherche universitaire est le résultat d'un choix, sous contraintes, celui de la rentabillité à très court terme et de l'élimination des investissements risqués comme la recherche à moyen terme.

(iii) Les facteurs limitatifs dus à la nature de la recherche universitaire et à l'évolution des universités:

Tout en reconnaissant que les universités ont leurs objectifs propres qui diffèrent de ceux des firmes, et que les chercheurs ont une priorité de publication, plusieurs entreprises déplorent que les universitaires soient difficiles à contrôler («les universitaires font ce qu'ils veulent» -... P2). Les réductions budgétaires, particulièrement draconiennes en Grande-Bretagne, qui ont obligé les universités à recourir à un financement contractuel, ont eu pour effet de faciliter les accords entre les universités et les entreprises. Mais la nouvelle donne a aussi des conséquences jugées négatives par les entreprises. Les universités seraient plus exigeantes en matière de contrats et de droits de propriété intellectuelle. Ainsi une entreprise (P15) a préféré recruter directement un scientifique pour la réalisation d'un projet plutôt que de passer un contrat, comprenant l'embauche d'un doctorant, avec l'University College of London (UCL), en raison du prix demandés7. Plusieurs entreprises citent également des négociations difficiles sur les brevets. Les conditions posées par le CNRS pour le dépôt d'un brevet en commun avec P12 sont considérées comme «dures» par cette

57. Nous avons vu que la contribution d'une entreprise au financement d'un postulant à un PhD, par ailleurs titulaire d'une bourse de l'EPSRC, est modeste (3000 livres pour l'étudiant et 1400 livres pour le département universitaire, chaque année). Mais UCL propose aussi des contrats «Full $\mathrm{PhD}$ » où, un financement équivalent à la bourse de l'EPSRC est également pris en charge par l'entreprise contractante. Le coût global (de l'ordre de 15000 livres annuellement) reste inférieur au salaire d'embauche d'un scientifique sur le marché. Cependant l'entreprise n'a pas la même maîtrise sur le iravail d'un doctorant dont elle a la cotutelle que sur celui d'un salarié qu'elle recrute. 
entreprise. Le partage des droits de propriété intellectuelle a été obtenu par $\mathrm{P} 13$ après une discussion avec UCL qui voulait disposer seule du brevet.

(iv) Les limitations liées à la capacité d'absorption des entreprises:

La difficulté à évaluer les connaissances disponibles dans les universités en raison de l'insuffisance de la formation universitaire des cadres de l'entreprise est explicitement avancée comme une cause limitative des contacts avec les universités. Une entreprise (P16) précise que les universités les plus faciles à approcher sont celles qui ont le plus besoin d'argent mais que cela n'indique pas quelles sont leurs compétences. Cette remarque vient d'une entreprise indé pendante; le manque de connaissances sur les recherches universitaires est confirmé par des entreprises qui ont peu de contacts avec le centre de recherche de leur groupe ( $\mathrm{P} 3)$. Dans le cas inverse, où il existe des relations suivies avec le laboratoire central, ce dernier peut pallier la faiblesse de la capacité d'évaluation de la recherche de l'unité de production $(\mathrm{P} 1)$. Ces dernières observations nous ramènent à la problématique sur les rapports entre recherche interne et recherche externe mise en œuvre par Cohen et Levinthal ainsi qu'à la limitation de l'application du concept d'absorption aux activités de recherche à long terme.

La place de la recherche universitaire - retour sur les hypothèses initiales

Une forte intensité d'innovation (ou de R-D) ne va pas systématiquement de pair avec des liens contractuels avec la recherche universitaire. Il est vrai que les tableaux de la section 4.1 . montrent que les liens forts avec les universités l'emportent pour l'ensemble constitué par les entreprises à forte et moyenne intensité de R-D (type II plus type III), alors que relations fortes et relations faibles s'équilibrent pour les entreprises de type I. Mais on trouve également des relations fortes avec les universités parmi les entreprises faiblement innovantes et inversement des liens faibles avec les universités parmi les firmes les plus innovantes. Par ailleurs, les entreprises de type II et de type III ont aussi des relations plus fortes avec les autres entreprises que les entreprises de type I. 
Sur onze entreprises ayant un niveau de R-D moyen ou élevé, sept ont des liens forts avec les universités. Parmi les quatre entreprises ayant de faibles liens avec les universités, deux (P1 et P7) n'ont pas de liens avec les autres entreprises. Il s'agit des filiales de très grands groupes, ayant des relations étroites avec les laboratoires centraux de leur groupe respectif et dont la majorité des produits est vendue à l'intérieur du groupe. En revanche, les deux autres entreprises (P10 et P15) entretiennent des relations très étroites avec leurs grands clients, et ont accès à leurs laboratoires. Ainsi, comme nous l'avons exposé initialement, une activité intensive de recherche et d'innovation peut être associée à des coopérations technologiques fortes avec les clients, sans qu'il existe nécessairement des relations étroites avec les universités.

Ces résultats, comme ceux qui ont été présentés à la section 4.1., sont compatibles avec le schéma initial postulant deux types de recherche et de collaborations associées et en conséquence la spécificité du recours à la recherche universitaire, mais la vue d'ensemble qui se dégage de l'enquête est plus nuancée et plus complexe. Pour les entreprises de l'échantillon il y a deux catégories de recherche, la R-D sur financement propre qui est liée aux objectifs à court terme, et la R-D à moyen et long terme qui dépend du financement externe. La recherche universitaire est incluse à la fois dans la recherche à court terme sur financement propre et dans la recherche à long terme subventionnée. La participation de la recherche universitaire à la recherche à court terme de l'entreprise prend la forme principale de prestations de services, tandis que sa contribution à la recherche à long terme se réalise essentiellement par la mise à disposition contractuelle de doctorants auprès des entreprises ${ }^{58}$. L'utilisation des services des universités est un peu plus fréquente chez les entreprises à faible intensité d'innovation, alors que l'embauche de doctorants est le fait des entreprises appartenant à un groupe doté d'un centre de recherche, à une exception près. Six entreprises bénéficient à la fois d'un contrat d'études militaires et du placement d'un doctorant, les deux principales sources de financement externe de la recherche à long terme. On peut, en effet, considérer que la mise à la disposition d'une entreprise d'un doctorant est une forme de subvention à la recherche, puisque l'État prend à sa charge la moitié (en France) ou plus (en Grande-Bretagne) de la rémunération du doctorant. Si

58. Six unités de production ont seulement des relations de services avec les universités (accès aux équipements et demande d'expertise), cinq établissements ont uniquement des contrats de recherche, tandis que cinq autres cumulent relations de services et contrats de recherche. 
l'accueil de doctorants ne se traduit pas toujours par une contribution directe et immédiate aux besoins de l'entreprise, l'embauche d'un doctorant est aussi un moyen d'accès aux autres ressources de l'université, y compris ses brevets. En effet, l'intérêt des entreprises pour les universités peut aussi être motivé par les brevets que celles-ci déposent. Le laboratoire central du groupe sert parfois d'intermédiaire entre les unités de production et les universités et peut sous cet aspect être considéré comme une source indirecte de connaissances universitaires. Il en va de même pour les laboratoires publics en France qui, fournisseurs de développements technologiques, puisent eux-mêmes dans les connaissances scientifiques accumulées par les laboratoires universitaires et du $\mathrm{CNRS}^{59}$. Les sociétés d'études et les laboratoires indépendants ne jouent qu'un rôle marginal comme fournisseurs de connaissances, en raison de leurs coûts plus élevés que ceux des universités et du retard qu'ils ont sur elles, dont ils sont dépendants pour les connaissances nouvelles. Cependant, une entreprise (P2) indique que la solution d'un problème qui se présente au cours du développement peut être sous-traitée simultanément auprès du laboratoire central du groupe, d'un laboratoire universitaire et d'un laboratoire indépendant. Enfin, il faut rappeler que tous les établissements n'accordent pas une place centrale aux objectifs technologiques. L'entreprise P2 préfère payer des consultants financiers plutôt que des consultants universitaires; les préoccupations de $\mathrm{P} 3$ ne sont pas technologiques mais centrées sur le management des ressources; l'entreprise P14 se dit plus intéressée par les programmes d'aides gouvernementaux qui ne visent pas spécifiquement la R-D, comme les subventions pour investissement et création d'emplois.

\section{L'impact des politiques publiques}

Des développements qui précèdent, on peut tirer une conséquence importante pour l'analyse des politiques d'innovation. L'incitation à la coopération ne passe pas, principalement, par les programmes spécifiques, gouvernementaux ou européens, mais par des dispositifs institutionnels dont ce n'est pas l'objectif premier, comme les marchés d'études militaires et la formation des doctorants par la recherche industrielle, sans oublier le

59. On rappellera que les transferts de connaissances des laboratoires du CNRS vers les entreprises ne se distinguent généralement pas des contributions des laboratoires universitaires, d'autant moins que, dans la plupart des cas, les laboratoires en contact avec les entreprises sont des unités mixtes CNRS-Université. 
164 L'économie du savoir: une économie de la collaboration?

rôle des appels d'offre du CNET en France, avant le début de sa privatisation.

Globalement, l'impact des programmes européens est faible: en 1997, seulement trois unités de production participent à un programme. Une entreprise ayant l'expérience d'une participation antérieure considère que ce fut un échec et d'autres n'envisagent pas d'être candidates. Le point de vue dominant est que les programmes européens concernent en priorité des entreprises de recherche. Cette appréciation générale doit être nuancée parce que, d'une part, des entreprises bénéficiant d'un contrat ont su en tirer des avantages qui ne se limitent pas à l'effet de subvention et, d'autre part, il faut tenir compte des contrats obtenus au niveau des centres de recherche des groupes. La participation à un projet du programme BRITEEuram a structuré toute l'activité de la firme P13, lui ouvrant des contacts avec des entreprises et des laboratoires de recherche. Selon les informations recueillies lors des entretiens au centre de R-D du groupe auquel appartient $\mathrm{P} 1$, il y a un engagement limité du centre technologique du groupe dans quelques collaborations pré-compétitives dans le cadre de programmes européens, sur des objectifs de partage des coûts et d'extension des droits de propriété.

L'incidence des programmes gouvernementaux n'est pas plus forte que celle des programmes européens, que ce soit en France ou en GrandeBretagne. La plus grande diversité des programmes britanniques ne se traduit pas par une proportion plus grande d'entreprises subventionnées au Royaume-Uni. Parmi les programmes britanniques, la formule des Teaching Company Scheme est proche de celle des thèses en entreprise, avec une participation financière de l'État (et d'agences régionales) à un projet commun à une entreprise et une université, la différence étant que le diplômé embauché n'a pas de thèse à préparer, et se trouve donc en principe plus disponible pour l'entreprise. Ce n'est pas l'avis de toutes les entreprises qui ont expérimenté ce dispositif (cf. 3.3).

Les réticences des entreprises à l'égard des programmes européens et gouvernementaux ont un fond commun, exprimé par un responsable de P2: "pour être utile, le financement public doit aider des projets décidés par l'entreprise». Ce qui n'exclut pas les projets de circonstances montés par des partenaires académiques et industriels, pour obtenir des financements publics. Le directeur d'un grand laboratoire de physique atomique et moléculaire, unité mixte CNRS-Université, consultant dans de grandes entreprises, dit avoir monté des projets communs, sans intérêt, avec des entreprises pour des raisons pécuniaires, dont un projet avec l'entreprise 
P6, «dont le contenu lui fait honte». Il ajoute que: «les entreprises sont surtout intéressées par les subventions, elles chassent les contrats avec financement public's.

L'emploi fréquent de doctorants ne peut pas s'expliquer par le seul intérêt pour les résultats des thèses ${ }^{60}$, et la modicité de l'investissement requis. Nous avons vu que les thèses industrielles avaient deux faces pour les entreprises: le domaine de recherche exploré par le doctorant et l'accès potentiel aux travaux, aux moyens, à l'environnement et aux relations du laboratoire universitaire d'accueil du thésard. L'emploi d'un doctorant est une des formes de constitution de la capacité d'évaluation des connaissances externes.

\section{Conclusion}

Nous avons fait le choix d'explorer les relations scientifiques et technologiques des entreprises avec leur environnement, plus particulièrement avec les institutions de la recherche publique, en prenant les unités de production comme poste d'observation. Cette option se trouve justifiée par l'association, quasi systématique dans chaque unité de production, d'une activité de recherche et développement avérée et de liens avec les universités. Il est à noter que la majorité des unités de production participent au financement d'un ou de plusieurs doctorants. La recherche se fait dans les unités de production et pas seulement dans les laboratoires centraux. L'intensité de la R-D se révèle être remarquablement élevée: la fourchette moyenne est comprise entre 9 et $13 \%$, et la moitié des six établissements qui se situent au-dessous ont une intensité de R-D au moins égale à $5 \%$, ce qui est loin d'être négligeable. Les réserves que l'on peut émettre quant au contenu des activités classées comme R-D, ou comme R$\mathrm{D}$ et études, ne sont pas différentes de celles qui sont applicables en général, à la mesure de ce type d'activités. Si notre étude avait été menée à partir d'informations recueillies auprès des centres de recherche des groupes (dont une partie a été visitée dans le cadre de l'enquête), la vision des collaborations des groupes aurait été sans doute plus globale. En revanche, nous n'aurions pas pu étudier la spécificité des échanges scientifiques et technologiques, avec les autres entreprises et avec la recherche universitaire ainsi que les complémentarités et substitutions, en

60. Au niveau des laboratoires centraux des groupes, l'intérêt pour les résultats spécifiques des thèses financées semble plus systématique. Voir J.-P. Beltramo, J.-J. Paul et C. Perret, «The recruitment of researchers...», op. cit. 
166 L'économie du savoir: une économie de la collaboration?

relation avec l'organisation de l'innovation, telles qu'elles ont été présentées à la section 4.1.

Notre démarche n'a pas été d'identifier les obstacles à un principe normatif de collaboration, mais d'examiner un de ses fondements implicites, l'intérêt des entreprises à bénéficier des connaissances universitaires. Alors que de nombreux auteurs considèrent que dans le domaine de la pharmacie, les résultats de la recherche, qu'ils proviennent des laboratoires universitaires ou des firmes de biotechnologie, réorientent en permanence les stratégies productives des multinationales, on peut soutenir, en s'en tenant, pour simplifier, au seul niveau des relations entre la recherche et la production, que les objectifs industriels des entreprises de l'optoélectronique déterminent leurs objectifs de recherche ${ }^{61}$.

Un des objectifs stratégiques de la majorité des entreprises de l'enquête, l'amélioration des procédés de production, oriente leurs activités de développements internes et se présente comme un facteur déterminant des relations avec la recherche universitaire. La complexité des procédés de production de beaucoup de composants et équipements optoélectroniques induit une forte demande potentielle à l'égard des universités, et, par ailleurs, la difficulté à établir des droits de propriété sur les procédés se présente comme un facteur de limitation des relations avec la recherche universitaire. La dichotomie mise en évidence, entre une recherche, permanente et autofinancée, pour résoudre les problèmes, et une recherche épisodique, mise en œuvre seulement si le financement extérieur est trouvé, illustre également le schéma de l'orientation de la recherche par la production. En sens inverse, la coopération avec la recherche universitaire semble avoir un faible impact sur la gestion interne de l'innovation, à l'exception du cas où cette coopération a son origine dans la participation de l'entreprise à un programme européen. La nature des relations des entreprises avec les universités, qui pour l'essentiel se traduisent par des relations de services et la cotutelle de doctorants, explique le peu d'incidence directe sur l'organisation de l'innovation à l'intérieur de la firme.

Nous n'avons pas de mesure quantitative des coopérations interentreprises, ni des coopérations entre les entreprises et les universités, comme le nombre ou le volume de contrats respectifs. Il apparaît ce-

61. C'est tout au moins le résultat qui domine dans nos enquêtes, sans que nous ayons l'intention de substituer une vision unilatérale à une autre. Voir sur les généralisations abusives: M. Trépanier et M.-P. Ippersiel, «Hiérarchie de la crédibilité et autonomie de la recherche - L'impensé des analyses des relations universités-entreprises", Actes de la recherche en sciences sociales, vol. 148, juin 2003, p. 74-82. 
pendant que les contacts sont plus fréquents et plus développés dans le cadre des relations clients/fournisseurs (avec réunions de travail régulières et accès aux laboratoires des partenaires) que dans les rapports avec les universités (les relations les plus suivies étant celles qui concernent certains doctorants et leurs responsables industriels). L'université conserve au regard des entreprises son rôle de pourvoyeur de recherche à long terme, en témoigne l'intérêt pour les doctorants, dont souvent les travaux n'entrent pas dans le cadre des préoccupations immédiates des unités de production. Mais les universités sont également sollicitées comme prestataires de services, pour deux raisons essentielles: les rémunérations demandées restent généralement faibles par rapport aux coût des services, malgré un surenchérissement suscité par la réduction des budgets publics des universités, et beaucoup d'expertises demandées sont liées à la recherche de pointe. C'est seulement dans ce cas, où le laboratoire universitaire se comporte en producteur de réponses à court terme, qu'il se substitue au laboratoire industriel. On peut d'ailleurs voir dans ce rôle de supplétif le résultat d'une carence de la recherche industrielle. Mais comme cela nous a été indiqué dans les entretiens avec les responsables des laboratoires publics, les universités ne peuvent pas remplacer les centres de transfert de technologie: elles les alimentent, en amont, de connaissances de base. Par ailleurs, alors que l'accent a parfois été mis sur le rôle nouveau des institutions de recherche indépendantes, comme productrices de connaissances, celles-ci n'apparaissent pratiquement pas dans notre enquête; leurs services sont jugés trop onéreux par rapport aux universités et leurs compétences, en retard sur celles des universitaires. En revanche, la plupart des entreprises françaises ont des liens très étroits avec les laboratoires publics, CEA-LETI et CNET.

Le démantèlement du CNET, institution qui représentait un exemple réussi de transfert de technologie, amène à s'interroger sur la possibilité de politiques scientifiques et technologiques visant à faciliter l'émergence d'une société de la connaissance, qui soient indépendantes des politiques destinées à faciliter la concurrence. Comme nous l'avons constaté, la règle de la concurrence continue à régir les rapports entre les entreprises dans le cadre des accords technologiques, mais cette concurrence par la coopération, en impliquant des acteurs de la recherche publique, a tendance à pénétrer le monde universitaire. Lors des entretiens dans les laboratoires universitaires et les laboratoires publics qui complétaient les enquêtes en entreprises, nous avons rencontré le cas, et il ne doit pas être rare, où deux équipes d'un même laboratoire universitaire, parce qu'elles avaient des 
partenaires industriels concurrents, étaient devenues rivales, avec les mêmes pratiques du secret que leurs alliés respectifs, alors qu'elles avaient un passé fructueux de collaboration. La recherche doit-elle se scinder en entreprises concurrentes? L'incitation à la collaboration semble une idée dépassée par les faits, la question n'est plus d'encourager la collaboration, mais de protéger l'identité et la fonction du partenaire public.

Jean-Paul BELTRAMO

Irédu-CNRS

Université de Bourgogne

\section{Résumé}

Analysant les caractéristiques de la demande de connaissances externes des entreprises en fonction de leurs régimes d'innovation, l'article s'intéresse plus particulièrement au rôle donné à la recherche universitaire dans les stratégies d'innovation des entreprises. Les résultats tirés d'enquêtes auprès d'entreprises britanniques et françaises, développant des composants optoélectroniques, mettent en évidence l'existence de deux catégories de recherche: la R-D sur financement propre qui est liée aux objectifs à court terme, et la R-D à moyen et long terme qui dépend du financement externe. La participation de la recherche universitaire à la recherche à court terme de l'entreprise prend la forme principale de prestations de services, tandis que sa contribution à la recherche à long terme se réalise essentiellement par la mise à disposition contractuelle de doctorants. De plus, les collaborations avec les universités ne semblent pas avoir d'effets directs sur l'organisation de l'innovation à l'intérieur de la firme.

Mots clés: Stratégies d'innovation, connaissances externes, collaborations de la recherche, relations universités-entreprises, optoélectronique, doctorant. 


\begin{abstract}
This paper aims to examine the features of the demand for external knowledge from the companies (according to their innovative regimes) and focus on the particular role of their relationships with academic research in their innovative strategies. The findings from the research visits to British and French establishments developing optoelectronic components, highlight two types of industrial research: self-funded short-term $R \& D$ on the one hand and external-funded medium or long-term $\mathrm{RD}$ on the other. To short-term R\&D in companies, universities contribute their technical expertises; to long-term $\mathrm{R} \& \mathrm{D}$, the resources of $\mathrm{PhD}$ students. Furthermore, it seems that the research collaboration with universities has no direct effect on the organisation of the innovative activities in the firms.
\end{abstract}

Keywords: Strategies for innovation, External knowledge, Research collaboration, Universities-enterprises relationships, Optoelectronics, $\mathrm{PhD}$ student.

\title{
Resumen
}

El objetivo de este artículo es analizar las características de la demanda de conocimientos externos de las empresas en función de sus regímenes de innovación, examinando en particular el papel dado a la investigación académica en sus estrategias de innovación. Los resultados extraídos de encuestas realizadas a empresas británicas y francesas, que desarrollan componentes opto-electrónicos, destacan la existencia de dos categorías de investigación: la R-D con financiación propia que está ligada a objetivos de corto plazo, y la R-D de medio y largo plazo que depende de financiación externa. La participación de la investigación universitaria en la investigación a corto plazo de la empresa se manifiesta principalmente a través de prestaciones de servicios, mientras que su contribución a la investigación a largo plazo se realiza esencialmente mediante contratos para doctorandos. Además, las colaboraciones con las universidades no parecen tener efectos directos sobre la organización de la innovación dentro de la empresa.

Palabras clave: Estrategias de innovación, conocimientos externos, colaboraciones de la investigación, relaciones universidad-empresas, optoelectrónica, doctorando 